\title{
A Models Approach to Analyzing and Forecasting Oil and Piracy in the Gulf of Guinea
}

\author{
Nana Raymond Lawrence Ofosu-Boateng \\ College of Transportation and Communications, Shanghai Maritime University, Shanghai, China \\ Email:boatnana@gmail.com
}

How to cite this paper: Ofosu-Boateng, N.R.L. (2017) A Models Approach to Analyzing and Forecasting Oil and Piracy in the Gulf of Guinea. Open Journal of Social Sciences, 5, 84-112. https://doi.org/10.4236/jss.2017.53009

Received: January 16, 2017

Accepted: March 14, 2017

Published: March 17, 2017

Copyright $\odot 2017$ by author and Scientific Research Publishing Inc. This work is licensed under the Creative Commons Attribution International License (CC BY 4.0).

http://creativecommons.org/licenses/by/4.0/ (c) (i) Open Access

\begin{abstract}
Piracy at sea is a great detriment to the transportation of goods through our seaways. Seaborne trade accounts for about $80 \%$ of global trade. To effectively monitor their territorial waters and Exclusive Economic Zones is problematic for many countries in West and Central Africa. To figure out the modus operandi of the pirates and be aware and proactive enough to create effective countermeasures remains a crucial endeavour to arrest this menace. The Gulf of Guinea (GoG) has been an active arena for piratical activities. This paper utilizes systematic analysis to determine and examine pirate incidents that occured from 2006 to 2015 with data from the International Maritime Bureau (IMB). Analysis of the data was done using RealStats resouce add-on for Excel and predictive modelling was used to test the forecasting ability of various models empirically. Three key models namely, Ordinal Logistic Regression, Bayesian Network Predictor, and Series Hazard Models are used to compute the probable increase in pirate attacks with a forecast into the next fifteen years. This is essential to the trend of the new wave of pirate attacks and its current and potential impact on Maritime Transportation and Maritime Security. While there is an international presence in the Gulf of Aden, there is no such dedicated force in the GoG. The findings from the statistical results are to assist policymakers in their decision making when using their scarce law enforcement apparatus to combat Maritime Piracy and their Maritime Transportation and Security challenges.
\end{abstract}

\section{Keywords}

Bayesian Network Predictor, Forecasting, Gulf of Guinea, Maritime Security, Maritime Transportation, Ordinal Logistic Regression, Series Hazard Model

\section{Introduction}

Prior to 2009, piracy appeared to be a limited problem and less of a menace to 
the international community. However, Hostis humani generis has been traditionally qualified due to the occasional surge of piracy activities. Since the 1990s, in particular, ships and their crew members have been held for ransom by small armed groups in territorial seas. The groups responsible for these activities claim to be protecting the fishing resources of Somalia [1]. At the time, these waters were pillaged by foreign fisherman and the coastal waters were used as a waste dumping ground because the government was unable to enforce the existing law. Establishing the rule of law was key to the proper functioning of the economy but according to [2], Somalia was greatly affected due to the breakdown in the rule of law with a ripple effect on its economy. These people were able to take advantage of the ineffective government, thereby increasing piracy within the Somalian waters as governmental attentions were focused elsewhere, such as political fights occurring on land. As a result of this inattention, piracy became bolder and became a significant menace beginning "from the Suez Canal and going through the Gulf of Aden to the narrow area between the Horn of Africa and the Arabian peninsula" [1]. Prima facie, the dynamics of piracy and armed robbery off the Somali coast can be attributed to the pivotal reason that Somaila is a failed state and thrives on the fortunes of piracy which is viewed as a lucrative business [3]. Three key elements must be present at one location for piracy to occur at sea. Firstly, there should be a pirate, a shipping vessel which is vulnerable, and good environmental conditions [4]. Piracy appears to be reoccurring in cycles. In the past this menace was defeated [5]. In the quest to normalise the shipping routes and keep them safe, a multinational force was deployed along the Horn of Africa corridor, with its associated resilience and challenges among the military personnel [6]. Within these areas, piracy has become increasingly advanced, partly due to technological advancements and partly due to increased daring by pirates, allowing ships to be attacked over a great distance. The limitations of their fibre glass skiffs due to their fuel capacity and speed made the pirates operate initially within a few nautical miles off the coastline [7]. Due to the increasing success in capturing ships and crews of ships, allowing for obtaining of ransom, pirates are once again the sub specie of Somali pirates-otherwise known as Hostes humani generis. International states have become increasingly alarmed due to the increased navigation danger through a significant choke-point of international traffic. Moreover, greater outrage has been induced due to pirate attacks on humanitarian ships, carrying supplies to the Somali people. There have been increasing incidences of piracy in these chokeholds due to governmental attention on other matters, such as political uprising or economic uncertainty. The Somalian coast, as explored in the following paragraph, is a valuable example of how piracy can occur even though governments are supposedly fighting the crime [1]. Ship owners and operators seek interest in the cost benefit and maritime safety of their ships through risk analysis when considering routes for their ships especially through pirate infested waters [8].

According to the Security Council, pirate activities off the Somalian coast are 
linked to threats to international peace and security. Resolution 733/1992 has allowed the Security Council to invoke Chapter VII in order to address the Somalian coast piracy situation, suggesting the threat to international peace and security. Moreover, it is suggested that the piracy activities threaten the peace and security of the Somalian region. Therefore, the Chinese Minister of Foreign Affairs approved Resolution 1851 in 2008 to decrease the threat to international peace and security-both on an international and a regional level. The goal of this action was to show that actions to fight "piracy off the Somali coasts be conducted within the framework of Chapter VII of the UN Charter". China has sought to play a greater role in its bid to rid the seas of the scourge of maritime piracy through peacekeeping and related counter piracy operations [1] [9]. Seaborne trade is very vulnerable to piracy and armed robbery and often presents as a potential target to attacks by terrorist. It is necessary to apply observation trends and lessons learned to curb this menace [1] [10].

Due to these attacks, there are increased concerns about the effectiveness of piracy laws which exist on an international level through the UN Convention on the Law of the Sea (also known as UNCLOS) in Articles 100 to 107, as well as 110. It is interesting to note that the UNCLOS articles are identical to the Geneva Convention of the High Seas of 1958 (Articles 14 to 22). Moreover, while some states are not bound by UNCLOS, they are bound by the Geneva Convention, suggesting that all articles in relation to sea law are the same or essentially the same. With a similarity to other crime forms, pirate attacks tend to cluster in space. If an attack occurs at one location, it heightens the risk of a similar act occurring at the same location or an area close to it [1] [11]. Yet, there are increasing concerns regarding the higher rates of piracy occurring in different areas of maritime waters despite the similarities in laws.

To the international community, Somalia remains an intractable challenge. The present state provides a fertile breeding ground for organized crime with sea piracy spear heading and disrupting maritime trade and security [12]. Devastating droughts coupled with a protracted civil war ushered in dire consequences bothering on humanitarian crisis [13]. Maritime security is two pronged, having an intrinsic and extrinsic dimension, which are threatened by sea piracy. The spill of saying an oil-laden ship by pirates spells an environmental disaster in the intrinsic dimension, while the direct users of the marine environment suffer in the extrinsic dimension [14].

\section{Research Problem, Questions, and Significance}

Piracy attacks in the Gulf of Guinea are increasing, even though armed robbery and piracy at the Gulf of Aden is on a gradual decline. According to [15], there was a significant increase of $30 \%$ in the acts of piracy between 2008 and 2013. In 2012 West African piracy attacks for the first time exceeded those off the Horn of Africa, with 966 sailors attacked in West Africa against 851 off the Somali Coast [16]. Combating piracy along the Gulf of Guinea (GoG) involves an extensive coastline which needs constant monitoring with the use of modern 
technologies as the Vessel Traffic System (VTS) [17]. The [18] [19] specifies that the Piracy Reporting Centre updated their records to 58 attacks by pirates, consisting of ten hijackings. With 11 attacks reported for the first quarter of 2013, and 27 attacks in 2012 (almost three times more than in 2011), the country Nigeria is seen as the most affected country. However, it is believed that other coastline countries and areas most affected apart from Nigeria in the Gulf of Guinea comprise Ghana, Togo, Benin and Bakassi. This is evident as pirates have increased their operations on the Ghanaian waters, which negatively affects maritime transportation and maritime security, and the recent discovery of oil will increase the risk of piracy on its high sea [20].

The research questions to be addressed include:

1) What are the causes of piracy in the Gulf of Guinea and obstacles to effective resolution?

2) What are the effects of oil piracy in the Gulf of Guinea on maritime transportation (concerning the effects on reputation damage to maritime transport company, insurance costs, transport price increase, extra cost on changing route, trade disruption and the loss of crude oil)?

3) What are the effects of oil piracy in the Gulf of Guinea on maritime security (considering the effects on security cost, more integration with the navy, dangers of sailing more nautical miles, threat to crew and lives, security equipment)?

4) What are the strategies required to manage these threats?

\subsection{Data Collection Criteria and Analysis}

The data used for this study will be obtained from the International Maritime Bureau and the IMB Piracy Reporting Centre. The time period being assessed is 2006 to 2015. The data will include all countries; however, particular emphasis will be placed on the Gulf of Guinea. The data indicator is the number of piracy incidences within the stated country. Therefore, the indicator is defined by actual attacks occurring globally. The data analysis was conducted using Real Stats add-in for Excel 2013 edition.

\subsection{Assumptions}

It is assumed that the data obtained from the IMB is accurate and up-to-date. It is assumed that the reporting person has been honest in the report and has provided all necessary information. It is assumed that all piracy attacks have been reported for the time period. Since the study is based on a quantitative method, it is assumed that the models used to forecast possible future piracy attacks are the most valid and flexible to meet the expectations of this particular study.

\subsection{Practical Significance}

The goal of this study is to provide ways that future piracy attacks can be predicted. In this respect, the purpose of the paper is to determine how many attacks can be expected in future time periods, which is beneficial in allowing governmental agencies ample opportunity to develop new tactics to prevent piracy 
or capture privateers. Through capturing privateers, it is possible that these individuals can shed light on reasons behind piracy. Moreover, with knowing the anticipated number of attacks, it is possible that governmental agencies can develop protections, such as an increased naval fleet, in order to protect ships that are operating in potentially dangerous waters.

\section{Ordinal Logistic Regression}

\subsection{Background}

The ordinal logistic regression is a statistics model that is commonly known as the ordered logit model or proportional odds model. This model is a regression model utilised for ordinal dependent variables. The model was established by Peter McCullagh. Within the model, it is assumed that if a survey's question is designed to be answered with a set choice (such as "poor" or "excellent") and the analysis's purpose is to determine the rate of prediction for a particular response based on responses to other questions, it is possible to use ordinal logistic regression [21] [22] [23] [24] [25]. Ordinal logistic regression can be considered as an extension of the initial logistic regression model, commonly applicable to dichotomous dependent variables. As a result, it is possible to analyse more than two ordered response categories.

The ordinal logistic regression model only applies to data meeting the proportional odds assumptions. The proportional odds assumptions assume that the proportions are divided by the response (such as "very poor", "poor", "good" "very good" or "excellent") into classifications, such as p1, p2, p3, p4 or p5, respectively. The logarithms of the odds, rather than the logarithms of the probabilities, of answering in a particular way. This is shown below:

$$
\begin{gathered}
\log \frac{p_{1}}{p_{2}+p_{3}+p_{4}+p_{5}}, 0 \\
\log \frac{p_{1}+p_{2}}{p_{3}+p_{4}+p_{5}}, 1 \\
\log \frac{p_{1}+p_{2}+p_{3}}{p_{4}+p_{5}}, 2 \\
\log \frac{p_{1}+p_{2}+p_{3}+p_{4}}{p_{5}}, 3
\end{gathered}
$$

Therefore, according to the proportional odds assumption is that the number added to each logarithm to obtain the next number is the same, allowing the logarithms to form an arithmetic sequence. According to the model, the number that exists in the last column, representing the number of required additions for the logarithm, is determined through a linear combination of observed variables [21] [22] [23] [24] [25]. The coefficients found in the linear combination cannot be estimated on a consistent basis through the ordinary least squares, resulting in a typical estimation by maximum likelihood. Maximum likelihood estimates are computed through iteratively reweighted least squares. Ordered response categories may be credit ratings (such as for bonds), Likert scales from opinion 
surveys, levels of spending (such as "high" or "low"), or employment status. A logistic regression model is used to analyze the ordinal outcome. In fitting a binary logistic regression model, there is a need to predict the probability of the outcome of interest by estimating a set of regression coefficients. The logistic model can be written in different ways. The version that shows what function of the probabilities' results in a linear combination of parameters and is as follows:

$$
\ln \left(\frac{\operatorname{Prob}(\text { event })}{(1-\operatorname{Prob}(\text { event })}\right)=\beta_{0}+\beta_{1} X_{1}+\cdots+\beta_{i} X_{i}
$$

In this equation, $\beta$ refers to the parameter (based on the data from Equation (1)). At the same time, $X$ refers to the number of occurrences. The logit refers to the left side of Equation (2). It refers to the log of the odds that an event occurs. This is calculated as the ratio of occurrences of an event based on the number that do not. The coefficients in the logistic regression model give how much the logit changes based on the values of the predictor variables.

\subsection{Application to the Data}

The data is shown (Table 1) for all countries and consider a specific time period. The range of occurrences is 0 to 160 . The coefficients will be based on 20 ranges.

Table 1. Ranges for ordinal logistic regression (Source; Adapted from [24] [26]).

\begin{tabular}{|c|c|c|}
\hline Range & Count & Ratio \\
\hline $\mathrm{P} 1=0-8$ & 552 & 0.8625 \\
\hline $\mathrm{P} 2=9-16$ & 51 & 0.0797 \\
\hline $\mathrm{P} 3=17-24$ & 9 & 0.0141 \\
\hline $\mathrm{P} 4=25-32$ & 8 & 0.0125 \\
\hline $\mathrm{P} 5=33-40$ & 4 & 0.0063 \\
\hline $\mathrm{P} 6=41-48$ & 4 & 0.0063 \\
\hline $\mathrm{P} 7=49-56$ & 3 & 0.0047 \\
\hline$P 8=57-64$ & 0 & 0.0000 \\
\hline $\mathrm{P} 9=65-72$ & 0 & 0.0000 \\
\hline$P 10=73-80$ & 1 & 0.0016 \\
\hline $\mathrm{P} 11=81-88$ & 1 & 0.0016 \\
\hline $\mathrm{P} 12=89-96$ & 1 & 0.0016 \\
\hline $\mathrm{P} 13=97-104$ & 1 & 0.0016 \\
\hline $\mathrm{P} 14=105-112$ & 2 & 0.0031 \\
\hline $\mathrm{P} 15=113-120$ & 1 & 0.0016 \\
\hline $\mathrm{P} 16=121-128$ & 0 & 0.0000 \\
\hline $\mathrm{P} 17=129-136$ & 0 & 0.0000 \\
\hline $\mathrm{P} 18=137-144$ & 1 & 0.0016 \\
\hline $\mathrm{P} 19=145-152$ & 0 & 0.0000 \\
\hline$P 20=153-160$ & 1 & 0.0016 \\
\hline
\end{tabular}


These are: $\mathrm{P} 1=0$ - 8; P2 = 9 - 16; P3 = 17 - 24; $\mathrm{P} 4=25$ - 32; $\mathrm{P} 5=33$ - 40; $\mathrm{P} 6=41$ $48 ; \mathrm{P} 7=49-56 ; \mathrm{P} 8=57-64 ; \mathrm{P} 9=65-72 ; \mathrm{P} 10=73-80 ; \mathrm{P} 11=81-88 ; \mathrm{P} 12=89-$ 96; P13 = 97 - 104; P14 = 105 - 112; P15 = 113 - 120; P16 = 121 - 128; P17 = 129 136; P18 = 137 - 144; P19 = 145 - 152; and P20 = 153 - 160.

The assumptions are defined as 0 to 19 (explained through Equation (1)) and are shown below (Table 2).

These are shown in the following Figure 1: (Source: Adapted from [24]).

Table 2. Ordinal logistic regression assumptions (Source: Adapted from [26]).

\begin{tabular}{|c|c|c|c|c|}
\hline Assumption & Numerator & Denominator & Result & Log (Result) \\
\hline 0 & 0.8625 & 0.1375 & 6.2727 & 0.7975 \\
\hline 1 & 0.9422 & 0.0578 & 16.2973 & 1.2121 \\
\hline 2 & 0.9563 & 0.0438 & 21.8571 & 1.3396 \\
\hline 3 & 0.9688 & 0.0313 & 31.0000 & 1.4914 \\
\hline 4 & 0.9750 & 0.0250 & 39.0000 & 1.5911 \\
\hline 5 & 0.9813 & 0.0188 & 52.3333 & 1.7188 \\
\hline 6 & 0.9859 & 0.0141 & 70.1111 & 1.8458 \\
\hline 7 & 0.9859 & 0.0141 & 70.1111 & 1.8458 \\
\hline 8 & 0.9859 & 0.0141 & 70.1111 & 1.8458 \\
\hline 9 & 0.9875 & 0.0125 & 79.0000 & 1.8976 \\
\hline 10 & 0.9891 & 0.0109 & 90.4286 & 1.9563 \\
\hline 11 & 0.9906 & 0.0094 & 105.6667 & 2.0239 \\
\hline 12 & 0.9922 & 0.0078 & 127.0000 & 2.1038 \\
\hline 13 & 0.9953 & 0.0047 & 212.3333 & 2.3270 \\
\hline 14 & 0.9969 & 0.0031 & 319.0000 & 2.5038 \\
\hline 15 & 0.9969 & 0.0031 & 319.0000 & 2.5038 \\
\hline 16 & 0.9969 & 0.0031 & 319.0000 & 2.5038 \\
\hline 17 & 0.9984 & 0.0016 & 639.0000 & 2.8055 \\
\hline 18 & 0.9984 & 0.0016 & 639.0000 & 2.8055 \\
\hline 19 & 1.0000 & 0.0016 & 640.0000 & 2.8062 \\
\hline
\end{tabular}

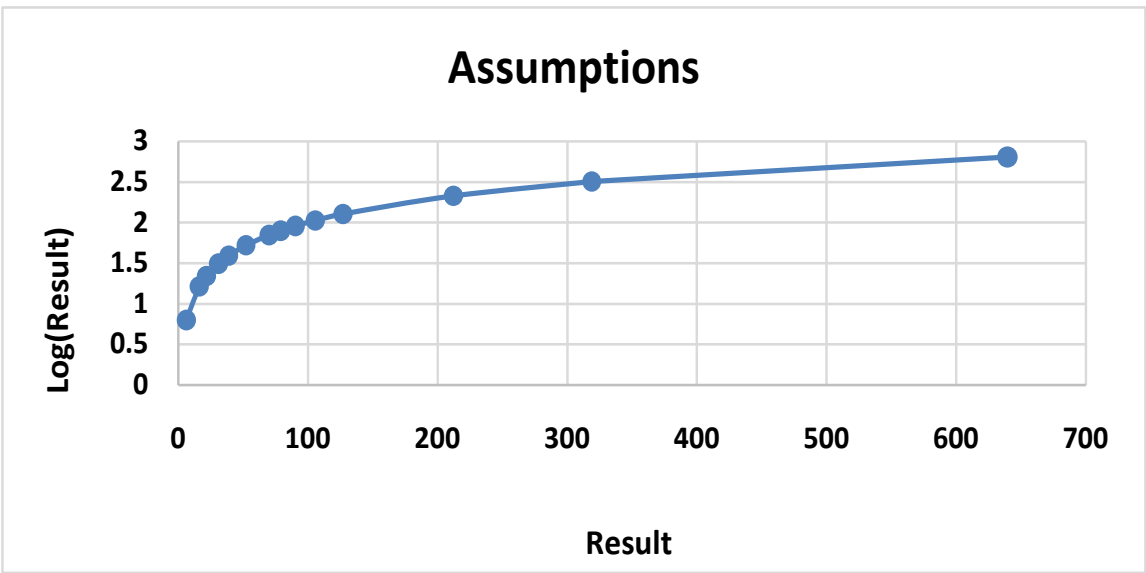

Figure 1. Assumptions for ordinal logistics model [24]. 
Based on the information from the preceding sections, the variable can be determined, as follows (Table 3).

When inputting the information from Table 2 [26] and the raw data from the Appendix, the following comparison can be seen for the binary logistic regression and the multimodal logistic regression.

The key takeaway from Table 4 [24] is that the results from the binary logistic regression is significant, as evident. At the same time, the multimodal logistic regression is significant, based on the $p$-value. The $\mathrm{R}$ Sq values were interesting to note as well. On a whole, the R Sq results for the binary logistic regression were higher than for the multimodal logistic regression. The whole of this information provides the ROC curve, shown in the following Table 5 and Figure 2.

The purpose of this model is to forecast the future anticipated attacks on the Gulf of Guinea. This is shown in the following Figure 3.

The purpose of Figure 3 [18] [19] is to show the number of piracy attacks within the areas around the Gulf of Guinea. These include Equatorial Guinea, Ghana, Guinea, and Guinea Bissau. It is noted that between 2006 and 2007, there was a sharp decline for both Ghana and Guinea in terms of total piracy attacks. This decline continued for Guinea, yet ended for Ghana. Moreover, between

Table 3. Ordinal logistics model variables (Source: Adapted from [26]).

\begin{tabular}{|c|c|c|c|c|}
\hline Variable & Logit & $\beta$ & $X$ & $\beta X$ \\
\hline 0 & & 0.7975 & & 0.7975 \\
\hline 1 & 669.0878 & 1.2121 & 552 & 669.0792 \\
\hline 2 & 68.3193 & 1.3396 & 51 & 68.3196 \\
\hline 3 & 13.4223 & 1.4914 & 9 & 13.4226 \\
\hline 4 & 12.7285 & 1.5911 & 8 & 12.7288 \\
\hline 5 & 6.8751 & 1.7188 & 4 & 6.8752 \\
\hline 6 & 7.3831 & 1.8458 & 4 & 7.3832 \\
\hline 7 & 5.5374 & 1.8458 & 3 & 5.5374 \\
\hline 8 & 0.0000 & 1.8458 & 0 & 0.0000 \\
\hline 9 & 0.0000 & 1.8976 & 0 & 0.0000 \\
\hline 10 & 1.9563 & 1.9563 & 1 & 1.9563 \\
\hline 11 & 2.0239 & 2.0239 & 1 & 2.0239 \\
\hline 12 & 2.1038 & 2.1038 & 1 & 2.1038 \\
\hline 13 & 2.3270 & 2.3270 & 1 & 2.3270 \\
\hline 14 & 5.0076 & 2.5038 & 2 & 5.0076 \\
\hline 15 & 2.5038 & 2.5038 & 1 & 2.5038 \\
\hline 16 & 0.0000 & 2.5038 & 0 & 0.0000 \\
\hline 17 & 0.0000 & 2.8055 & 0 & 0.0000 \\
\hline 18 & 2.8055 & 2.8055 & 1 & 2.8055 \\
\hline 19 & 0.0000 & 2.8062 & 0 & 0.0000 \\
\hline$\Sigma$ & & & & 802.0814 \\
\hline
\end{tabular}


Table 4. Binary logistic regression vs. multimodal logistic regression (Source: Adapted from [24].

\begin{tabular}{|c|c|c|c|c|c|}
\hline \multicolumn{3}{|c|}{ Binary Logistic Regression } & \multicolumn{3}{|c|}{ Multimodal Logistic Regression } \\
\hline Coefficient & LLO & -1445.4 & Coefficient & LL & -1160.2 \\
\hline-2.7826 & LL1 & -1365.7 & -2.7826 & LLO & -1183.4 \\
\hline-0.0010 & & & -0.0010 & & \\
\hline-0.0611 & Chi-Sq & 159.4202 & -0.0611 & Chi-sq & 84.4491 \\
\hline 0.0788 & df & 9 & 0.0788 & df & 8 \\
\hline-0.1077 & $p$-value & 0.0000 & -0.1077 & $p$-value & 0.0000 \\
\hline 0.0229 & alpha & 0.05 & 0.0229 & & \\
\hline 0.0416 & sig & yes & 0.0416 & & \\
\hline-0.1509 & & & -0.1509 & & \\
\hline 0.1606 & R-Sq (L) & 0.0551 & 0.1606 & R-sq (L) & 0.0021 \\
\hline \multirow[t]{7}{*}{0.0506} & $\mathrm{R}-\mathrm{Sq}(\mathrm{CS})$ & 0.0261 & 0.0506 & R-sq (CS) & 0.0089 \\
\hline & $\mathrm{R}-\mathrm{Sq}(\mathrm{N})$ & 0.0685 & & R-sq (N) & 0.0090 \\
\hline & Hosmer & 93.3277 & & & \\
\hline & df & 62 & & & \\
\hline & $p$-value & 0.0062 & & & \\
\hline & alpha & 0.05 & & & \\
\hline & sig & yes & & & \\
\hline
\end{tabular}

Table 5. ROC curve (Source: Adapted from [24]).

\begin{tabular}{cccccccc}
\hline p-Pred & Failure & Success & Fail-Cum & Suc-Cum & FPR & TPR & AUC \\
\hline 0.0003 & 498 & 0 & 498 & 0 & 1.0000 & 1.0000 & 0.0882 \\
0.0009 & 345 & 0 & 843 & 0 & 0.9118 & 1.0000 & 0.0611 \\
0.0155 & 49 & 2 & 892 & 2 & 0.8507 & 1.0000 & 0.0087 \\
0.0239 & 34 & 0 & 926 & 2 & 0.8360 & 0.9949 & 0.0173 \\
0.0255 & 98 & 0 & 1024 & 2 & 0.8186 & 0.9949 & 0.0051 \\
0.0325 & 29 & 3 & 1053 & 5 & 0.8135 & 0.9872 & 0.0033 \\
0.0362 & 19 & 2 & 1072 & 7 & 0.8101 & 0.9821 & 0.0059 \\
0.0362 & 34 & 5 & 1106 & 12 & 0.8041 & 0.9692 & 0.0017 \\
0.0372 & 10 & 0 & 1116 & 12 & 0.8023 & 0.9692 & 0.0014 \\
0.0427 & 8 & 0 & 1124 & 12 & 0.8009 & 0.9692 & 0.0070 \\
0.0430 & 41 & 9 & 1165 & 21 & 0.7936 & 0.9462 & 0.0015 \\
0.0435 & 9 & 0 & 1174 & 21 & 0.7920 & 0.9462 & 0.0017 \\
0.0486 & 10 & 1 & 1184 & 22 & 0.7903 & 0.9436 & 0.0074 \\
0.0490 & 44 & 0 & 1228 & 22 & 0.7825 & 0.9436 & 0.0010 \\
0.0498 & 6 & 0 & 1234 & 22 & 0.7814 & 0.9436 & 0.0017 \\
0.0504 & 10 & 4 & 1244 & 26 & 0.7796 & 0.9333 & 0.0023 \\
0.0505 & 14 & 1 & 1258 & 27 & 0.7771 & 0.9308 & 0.0020 \\
0.0509 & 12 & 2 & 1270 & 29 & 0.7750 & 0.9256 & 0.0007 \\
0.0518 & 4 & 0 & 1274 & 29 & 0.7743 & 0.9256 & 0.0003 \\
0.0519 & 2 & 0 & 1276 & 29 & 0.7740 & 0.9256 & 0.0002 \\
\hline
\end{tabular}


N. R. L. Ofosu-Boateng

Continued

\begin{tabular}{|c|c|c|c|c|c|c|c|}
\hline 0.0526 & 1 & 0 & 1277 & 29 & 0.7738 & 0.9256 & 0.0002 \\
\hline 0.0526 & 1 & 0 & 1278 & 29 & 0.7736 & 0.9256 & 0.0002 \\
\hline 0.0526 & 1 & 0 & 1279 & 29 & 0.7734 & 0.9256 & 0.0002 \\
\hline 0.0526 & 1 & 0 & 1280 & 29 & 0.7733 & 0.9256 & 0.0010 \\
\hline 0.0541 & 6 & 0 & 1286 & 29 & 0.7722 & 0.9256 & 0.0007 \\
\hline 0.0549 & 4 & 0 & 1290 & 29 & 0.7715 & 0.9256 & 0.0002 \\
\hline 0.0550 & 1 & 0 & 1291 & 29 & 0.7713 & 0.9256 & 0.0002 \\
\hline 0.0550 & 1 & 0 & 1292 & 29 & 0.7711 & 0.9256 & 0.0002 \\
\hline 0.0550 & 1 & 0 & 1293 & 29 & 0.7709 & 0.9256 & 0.0002 \\
\hline 0.0550 & 1 & 0 & 1294 & 29 & 0.7708 & 0.9256 & 0.0002 \\
\hline 0.0550 & 1 & 0 & 1295 & 29 & 0.7706 & 0.9256 & 0.0002 \\
\hline 0.0582 & 1 & 0 & 1296 & 29 & 0.7704 & 0.9256 & 0.0002 \\
\hline 0.0582 & 1 & 0 & 1297 & 29 & 0.7702 & 0.9256 & 0.0002 \\
\hline 0.0582 & 1 & 0 & 1298 & 29 & 0.7701 & 0.9256 & 0.0002 \\
\hline 0.0582 & 1 & 0 & 1299 & 29 & 0.7699 & 0.9256 & 0.0043 \\
\hline 0.0588 & 26 & 1 & 1325 & 30 & 0.7653 & 0.9231 & 0.0005 \\
\hline 0.0590 & 3 & 0 & 1328 & 30 & 0.7647 & 0.9231 & 0.0016 \\
\hline 0.0592 & 10 & 1 & 1338 & 31 & 0.7630 & 0.9205 & 0.0049 \\
\hline 0.0606 & 30 & 0 & 1368 & 31 & 0.7577 & 0.9205 & 0.0018 \\
\hline 0.0607 & 11 & 1 & 1379 & 32 & 0.7557 & 0.9179 & 0.0111 \\
\hline 0.0615 & 68 & 0 & 1447 & 32 & 0.7437 & 0.9179 & 0.0002 \\
\hline 0.0627 & 1 & 0 & 1448 & 32 & 0.7435 & 0.9179 & 0.0002 \\
\hline 0.0627 & 1 & 0 & 1449 & 32 & 0.7433 & 0.9179 & 0.0002 \\
\hline 0.0627 & 1 & 0 & 1450 & 32 & 0.7431 & 0.9179 & 0.0010 \\
\hline 0.0635 & 6 & 2 & 1456 & 34 & 0.7421 & 0.9128 & 0.0036 \\
\hline 0.0650 & 22 & 1 & 1478 & 35 & 0.7382 & 0.9103 & 0.0390 \\
\hline 0.0650 & 242 & 14 & 1720 & 49 & 0.6953 & 0.8744 & 0.0033 \\
\hline 0.0663 & 21 & 0 & 1741 & 49 & 0.6916 & 0.8744 & 0.0014 \\
\hline 0.0663 & 9 & 0 & 1750 & 49 & 0.6900 & 0.8744 & 0.0019 \\
\hline 0.0672 & 12 & 0 & 1762 & 49 & 0.6879 & 0.8744 & 0.0003 \\
\hline 0.0677 & 2 & 0 & 1764 & 49 & 0.6875 & 0.8744 & 0.0014 \\
\hline 0.0693 & 9 & 0 & 1773 & 49 & 0.6859 & 0.8744 & 0.0037 \\
\hline 0.0698 & 24 & 1 & 1797 & 50 & 0.6817 & 0.8718 & 0.0006 \\
\hline 0.0722 & 4 & 0 & 1801 & 50 & 0.6810 & 0.8718 & 0.0053 \\
\hline 0.0732 & 34 & 5 & 1835 & 55 & 0.6749 & 0.8590 & 0.4764 \\
\hline 0.0735 & 3131 & 249 & 4966 & 304 & 0.1203 & 0.2205 & 0.0010 \\
\hline 0.0777 & 25 & 2 & 4991 & 306 & 0.1159 & 0.2154 & 0.0069 \\
\hline 0.0784 & 180 & 11 & 5171 & 317 & 0.0840 & 0.1872 & 0.0008 \\
\hline 0.0862 & 23 & 0 & 5194 & 317 & 0.0799 & 0.1872 & 0.0045 \\
\hline 0.0870 & 137 & 13 & 5331 & 330 & 0.0556 & 0.1538 & 0.0014 \\
\hline 0.1134 & 53 & 11 & 5384 & 341 & 0.0462 & 0.1256 & 0.0015 \\
\hline 0.1196 & 69 & 9 & 5453 & 350 & 0.0340 & 0.1026 & 0.0018 \\
\hline 0.1300 & 97 & 13 & 5550 & 363 & 0.0168 & 0.0692 & 0.0012 \\
\hline \multirow[t]{2}{*}{0.1994} & 95 & 27 & 5645 & 390 & 0.0000 & 0.0000 & 0.0000 \\
\hline & & & & & & & 0.8095 \\
\hline
\end{tabular}




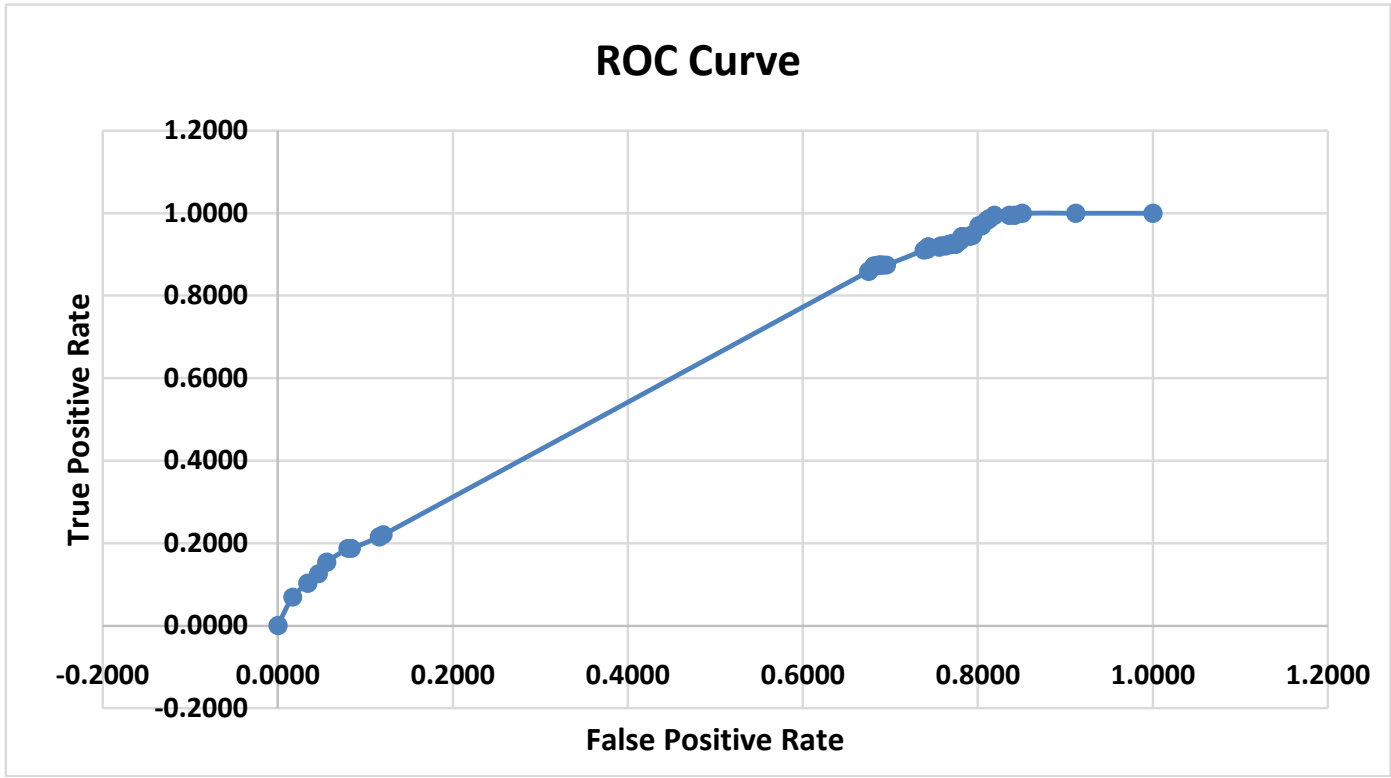

Figure 2. ROC model (Source: Adapted from [24]).

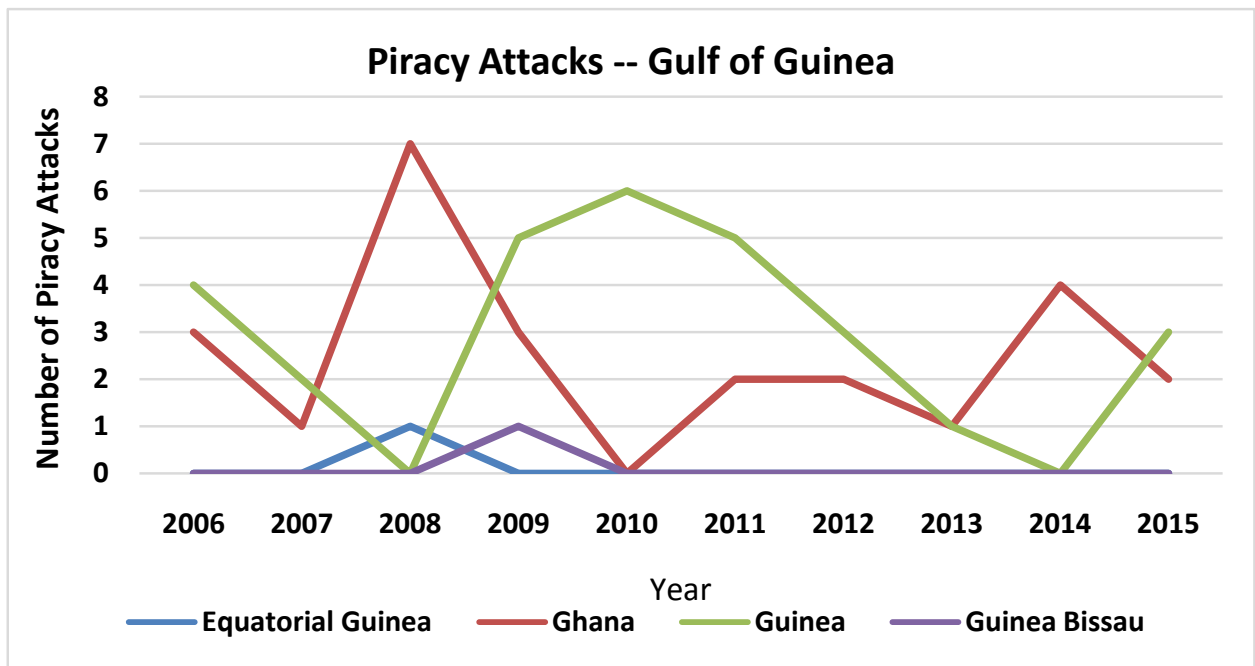

Figure 3. Piracy attacks on the gulf of Guinea (Source: [18] [19]).

2007 and 2008, there was an increase in piracy attacks in Equatorial Guinea. The most number of attacks occurred in 2008 in Ghana, followed by 2010 in Equatorial Guinea, before experiencing a decrease for most areas. Following the spike, Ghana's rate of piracy attacks decreased significantly, temporarily increasing in 2014. Within Equatorial Guinea, the spike decreased significantly, then increased again. Based on the ordinal logistic regression model, it is possible to predict the number of attacks. This is shown below (Table 6).

Based on this information, it can be assumed that piracy attacks will increase/decrease by approximately 5 per year. This can be seen forecasted from 2016 to 2030 for high and low. For 2016, the values are based on 2015. For 2017 and after, the values are based on the previous forecasted attacks, as shown in the Table 7 . The graph Figure 4 only shows the total forecasted attacks. 
Table 6. Gulf of Guinea piracy attack predictions based on ordinal logistic regression model (Source: Author's compilation from [18] [19]).

\begin{tabular}{|c|c|c|c|c|c|c|c|c|c|c|}
\hline Location & 06 & 07 & 08 & 09 & 10 & 11 & 12 & 13 & 14 & 15 \\
\hline Equatorial Guinea & 0 & 0 & 1 & 0 & 0 & 0 & 0 & 0 & 0 & 0 \\
\hline Ghana & 3 & 1 & 7 & 3 & 0 & 2 & 2 & 1 & 4 & 2 \\
\hline Guinea & 4 & 2 & 0 & 5 & 6 & 5 & 3 & 1 & 0 & 3 \\
\hline Guinea Bissau & 0 & 0 & 0 & 1 & 0 & 0 & 0 & 0 & 0 & 0 \\
\hline \multirow[t]{3}{*}{ Total } & 7 & 3 & 8 & 9 & 6 & 7 & 5 & 2 & 4 & 5 \\
\hline & \multicolumn{10}{|c|}{ Equatorial Guinea } \\
\hline & 0 & 0 & 1 & 0 & 0 & 0 & 0 & 0 & 0 & 0 \\
\hline 0.7975 & 0.0 & 0.0 & 0.8 & 0.0 & 0.0 & 0.0 & 0.0 & 0.0 & 0.0 & 0.0 \\
\hline 1.2121 & 0.0 & 0.0 & 1.2 & 0.0 & 0.0 & 0.0 & 0.0 & 0.0 & 0.0 & 0.0 \\
\hline 1.3396 & 0.0 & 0.0 & 1.3 & 0.0 & 0.0 & 0.0 & 0.0 & 0.0 & 0.0 & 0.0 \\
\hline 1.4914 & 0.0 & 0.0 & 1.5 & 0.0 & 0.0 & 0.0 & 0.0 & 0.0 & 0.0 & 0.0 \\
\hline 1.5911 & 0.0 & 0.0 & 1.6 & 0.0 & 0.0 & 0.0 & 0.0 & 0.0 & 0.0 & 0.0 \\
\hline 1.7188 & 0.0 & 0.0 & 1.7 & 0.0 & 0.0 & 0.0 & 0.0 & 0.0 & 0.0 & 0.0 \\
\hline 1.8458 & 0.0 & 0.0 & 1.8 & 0.0 & 0.0 & 0.0 & 0.0 & 0.0 & 0.0 & 0.0 \\
\hline 1.8458 & 0.0 & 0.0 & 1.8 & 0.0 & 0.0 & 0.0 & 0.0 & 0.0 & 0.0 & 0.0 \\
\hline 1.8458 & 0.0 & 0.0 & 1.8 & 0.0 & 0.0 & 0.0 & 0.0 & 0.0 & 0.0 & 0.0 \\
\hline 1.8976 & 0.0 & 0.0 & 1.9 & 0.0 & 0.0 & 0.0 & 0.0 & 0.0 & 0.0 & 0.0 \\
\hline 1.9563 & 0.0 & 0.0 & 2.0 & 0.0 & 0.0 & 0.0 & 0.0 & 0.0 & 0.0 & 0.0 \\
\hline 2.0239 & 0.0 & 0.0 & 2.0 & 0.0 & 0.0 & 0.0 & 0.0 & 0.0 & 0.0 & 0.0 \\
\hline 2.1038 & 0.0 & 0.0 & 2.1 & 0.0 & 0.0 & 0.0 & 0.0 & 0.0 & 0.0 & 0.0 \\
\hline 2.3270 & 0.0 & 0.0 & 2.3 & 0.0 & 0.0 & 0.0 & 0.0 & 0.0 & 0.0 & 0.0 \\
\hline 2.5038 & 0.0 & 0.0 & 2.5 & 0.0 & 0.0 & 0.0 & 0.0 & 0.0 & 0.0 & 0.0 \\
\hline 2.5038 & 0.0 & 0.0 & 2.5 & 0.0 & 0.0 & 0.0 & 0.0 & 0.0 & 0.0 & 0.0 \\
\hline 2.5038 & 0.0 & 0.0 & 2.5 & 0.0 & 0.0 & 0.0 & 0.0 & 0.0 & 0.0 & 0.0 \\
\hline 2.8055 & 0.0 & 0.0 & 2.8 & 0.0 & 0.0 & 0.0 & 0.0 & 0.0 & 0.0 & 0.0 \\
\hline 2.8055 & 0.0 & 0.0 & 2.8 & 0.0 & 0.0 & 0.0 & 0.0 & 0.0 & 0.0 & 0.0 \\
\hline 2.8062 & 0.0 & 0.0 & 2.8 & 0.0 & 0.0 & 0.0 & 0.0 & 0.0 & 0.0 & 0.0 \\
\hline \multirow[t]{3}{*}{ Total } & 0.0 & 0.0 & 39.9 & 0.0 & 0.0 & 0.0 & 0.0 & 0.0 & 0.0 & 0.0 \\
\hline & \multicolumn{10}{|c|}{ Ghana } \\
\hline & 3 & 1 & 7 & 3 & 0 & 2 & 2 & 1 & 4 & 2 \\
\hline 0.7975 & 2.4 & 0.8 & 5.6 & 2.4 & 0.0 & 1.6 & 1.6 & 0.8 & 3.2 & 1.6 \\
\hline 1.2121 & 3.6 & 1.2 & 8.5 & 3.6 & 0.0 & 2.4 & 2.4 & 1.2 & 4.8 & 2.4 \\
\hline 1.3396 & 4.0 & 1.3 & 9.4 & 4.0 & 0.0 & 2.7 & 2.7 & 1.3 & 5.4 & 2.7 \\
\hline 1.4914 & 4.5 & 1.5 & 10.4 & 4.5 & 0.0 & 3.0 & 3.0 & 1.5 & 6.0 & 3.0 \\
\hline 1.5911 & 4.8 & 1.6 & 11.1 & 4.8 & 0.0 & 3.2 & 3.2 & 1.6 & 6.4 & 3.2 \\
\hline 1.7188 & 5.2 & 1.7 & 12.0 & 5.2 & 0.0 & 3.4 & 3.4 & 1.7 & 6.9 & 3.4 \\
\hline 1.8458 & 5.5 & 1.8 & 12.9 & 5.5 & 0.0 & 3.7 & 3.7 & 1.8 & 7.4 & 3.7 \\
\hline 1.8458 & 5.5 & 1.8 & 12.9 & 5.5 & 0.0 & 3.7 & 3.7 & 1.8 & 7.4 & 3.7 \\
\hline 1.8458 & 5.5 & 1.8 & 12.9 & 5.5 & 0.0 & 3.7 & 3.7 & 1.8 & 7.4 & 3.7 \\
\hline
\end{tabular}




\section{Continued}

\begin{tabular}{|c|c|c|c|c|c|c|c|c|c|c|}
\hline 1.8976 & 5.7 & 1.9 & 13.3 & 5.7 & 0.0 & 3.8 & 3.8 & 1.9 & 7.6 & 3.8 \\
\hline 1.9563 & 5.9 & 2.0 & 13.7 & 5.9 & 0.0 & 3.9 & 3.9 & 2.0 & 7.8 & 3.9 \\
\hline 2.0239 & 6.1 & 2.0 & 14.2 & 6.1 & 0.0 & 4.0 & 4.0 & 2.0 & 8.1 & 4.0 \\
\hline 2.1038 & 6.3 & 2.1 & 14.7 & 6.3 & 0.0 & 4.2 & 4.2 & 2.1 & 8.4 & 4.2 \\
\hline 2.3270 & 7.0 & 2.3 & 16.3 & 7.0 & 0.0 & 4.7 & 4.7 & 2.3 & 9.3 & 4.7 \\
\hline 2.5038 & 7.5 & 2.5 & 17.5 & 7.5 & 0.0 & 5.0 & 5.0 & 2.5 & 10.0 & 5.0 \\
\hline 2.5038 & 7.5 & 2.5 & 17.5 & 7.5 & 0.0 & 5.0 & 5.0 & 2.5 & 10.0 & 5.0 \\
\hline 2.5038 & 7.5 & 2.5 & 17.5 & 7.5 & 0.0 & 5.0 & 5.0 & 2.5 & 10.0 & 5.0 \\
\hline 2.8055 & 8.4 & 2.8 & 19.6 & 8.4 & 0.0 & 5.6 & 5.6 & 2.8 & 11.2 & 5.6 \\
\hline 2.8055 & 8.4 & 2.8 & 19.6 & 8.4 & 0.0 & 5.6 & 5.6 & 2.8 & 11.2 & 5.6 \\
\hline 2.8062 & 8.4 & 2.8 & 19.6 & 8.4 & 0.0 & 5.6 & 5.6 & 2.8 & 11.2 & 5.6 \\
\hline \multirow[t]{3}{*}{ Total } & 119.8 & 39.9 & 279.5 & 119.8 & 0.0 & 79.8 & 79.8 & 39.9 & 159.7 & 79.8 \\
\hline & \multicolumn{10}{|c|}{ Guinea } \\
\hline & 4 & 2 & 0 & 5 & 6 & 5 & 3 & 1 & 0 & 3 \\
\hline 0.7975 & 3.2 & 1.6 & 0.0 & 4.0 & 4.8 & 4.0 & 2.4 & 0.8 & 3.2 & 2.4 \\
\hline 1.2121 & 4.8 & 2.4 & 0.0 & 6.1 & 7.3 & 6.1 & 3.6 & 1.2 & 4.8 & 3.6 \\
\hline 1.3396 & 5.4 & 2.7 & 0.0 & 6.7 & 8.0 & 6.7 & 4.0 & 1.3 & 5.4 & 4.0 \\
\hline 1.4914 & 6.0 & 3.0 & 0.0 & 7.5 & 8.9 & 7.5 & 4.5 & 1.5 & 6.0 & 4.5 \\
\hline 1.5911 & 6.4 & 3.2 & 0.0 & 8.0 & 9.5 & 8.0 & 4.8 & 1.6 & 6.4 & 4.8 \\
\hline 1.7188 & 6.9 & 3.4 & 0.0 & 8.6 & 10.3 & 8.6 & 5.2 & 1.7 & 6.9 & 5.2 \\
\hline 1.8458 & 7.4 & 3.7 & 0.0 & 9.2 & 11.1 & 9.2 & 5.5 & 1.8 & 7.4 & 5.5 \\
\hline 1.8458 & 7.4 & 3.7 & 0.0 & 9.2 & 11.1 & 9.2 & 5.5 & 1.8 & 7.4 & 5.5 \\
\hline 1.8458 & 7.4 & 3.7 & 0.0 & 9.2 & 11.1 & 9.2 & 5.5 & 1.8 & 7.4 & 5.5 \\
\hline 1.8976 & 7.6 & 3.8 & 0.0 & 9.5 & 11.4 & 9.5 & 5.7 & 1.9 & 7.6 & 5.7 \\
\hline 1.9563 & 7.8 & 3.9 & 0.0 & 9.8 & 11.7 & 9.8 & 5.9 & 2.0 & 7.8 & 5.9 \\
\hline 2.0239 & 8.1 & 4.0 & 0.0 & 10.1 & 12.1 & 10.1 & 6.1 & 2.0 & 8.1 & 6.1 \\
\hline 2.1038 & 8.4 & 4.2 & 0.0 & 10.5 & 12.6 & 10.5 & 6.3 & 2.1 & 8.4 & 6.3 \\
\hline 2.3270 & 9.3 & 4.7 & 0.0 & 11.6 & 14.0 & 11.6 & 7.0 & 2.3 & 9.3 & 7.0 \\
\hline 2.5038 & 10.0 & 5.0 & 0.0 & 12.5 & 15.0 & 12.5 & 7.5 & 2.5 & 10.0 & 7.5 \\
\hline 2.5038 & 10.0 & 5.0 & 0.0 & 12.5 & 15.0 & 12.5 & 7.5 & 2.5 & 10.0 & 7.5 \\
\hline 2.5038 & 10.0 & 5.0 & 0.0 & 12.5 & 15.0 & 12.5 & 7.5 & 2.5 & 10.0 & 7.5 \\
\hline 2.8055 & 11.2 & 5.6 & 0.0 & 14.0 & 16.8 & 14.0 & 8.4 & 2.8 & 11.2 & 8.4 \\
\hline 2.8055 & 11.2 & 5.6 & 0.0 & 14.0 & 16.8 & 14.0 & 8.4 & 2.8 & 11.2 & 8.4 \\
\hline 2.8062 & 11.2 & 5.6 & 0.0 & 14.0 & 16.8 & 14.0 & 8.4 & 2.8 & 11.2 & 8.4 \\
\hline \multirow[t]{3}{*}{ Total } & 159.7 & 79.8 & 0.0 & 199.6 & 239.5 & 199.6 & 119.8 & 39.9 & 159.7 & 119.8 \\
\hline & \multicolumn{10}{|c|}{ Guinea Bissau } \\
\hline & 0 & 0 & 0 & 1 & 0 & 0 & 0 & 0 & 0 & 0 \\
\hline 0.7975 & 0.0 & 0.0 & 0.0 & 0.8 & 0.0 & 0.0 & 0.0 & 0.0 & 0.0 & 0.0 \\
\hline 1.2121 & 0.0 & 0.0 & 0.0 & 1.2 & 0.0 & 0.0 & 0.0 & 0.0 & 0.0 & 0.0 \\
\hline 1.3396 & 0.0 & 0.0 & 0.0 & 1.3 & 0.0 & 0.0 & 0.0 & 0.0 & 0.0 & 0.0 \\
\hline 1.4914 & 0.0 & 0.0 & 0.0 & 1.5 & 0.0 & 0.0 & 0.0 & 0.0 & 0.0 & 0.0 \\
\hline
\end{tabular}




\begin{tabular}{|c|c|c|c|c|c|c|c|c|c|c|}
\hline 1.5911 & 0.0 & 0.0 & 0.0 & 1.6 & 0.0 & 0.0 & 0.0 & 0.0 & 0.0 & 0.0 \\
\hline 1.7188 & 0.0 & 0.0 & 0.0 & 1.7 & 0.0 & 0.0 & 0.0 & 0.0 & 0.0 & 0.0 \\
\hline 1.8458 & 0.0 & 0.0 & 0.0 & 1.8 & 0.0 & 0.0 & 0.0 & 0.0 & 0.0 & 0.0 \\
\hline 1.8458 & 0.0 & 0.0 & 0.0 & 1.8 & 0.0 & 0.0 & 0.0 & 0.0 & 0.0 & 0.0 \\
\hline 1.8458 & 0.0 & 0.0 & 0.0 & 1.8 & 0.0 & 0.0 & 0.0 & 0.0 & 0.0 & 0.0 \\
\hline 1.8976 & 0.0 & 0.0 & 0.0 & 1.9 & 0.0 & 0.0 & 0.0 & 0.0 & 0.0 & 0.0 \\
\hline 1.9563 & 0.0 & 0.0 & 0.0 & 2.0 & 0.0 & 0.0 & 0.0 & 0.0 & 0.0 & 0.0 \\
\hline 2.0239 & 0.0 & 0.0 & 0.0 & 2.0 & 0.0 & 0.0 & 0.0 & 0.0 & 0.0 & 0.0 \\
\hline 2.1038 & 0.0 & 0.0 & 0.0 & 2.1 & 0.0 & 0.0 & 0.0 & 0.0 & 0.0 & 0.0 \\
\hline 2.3270 & 0.0 & 0.0 & 0.0 & 2.3 & 0.0 & 0.0 & 0.0 & 0.0 & 0.0 & 0.0 \\
\hline 2.5038 & 0.0 & 0.0 & 0.0 & 2.5 & 0.0 & 0.0 & 0.0 & 0.0 & 0.0 & 0.0 \\
\hline 2.5038 & 0.0 & 0.0 & 0.0 & 2.5 & 0.0 & 0.0 & 0.0 & 0.0 & 0.0 & 0.0 \\
\hline 2.5038 & 0.0 & 0.0 & 0.0 & 2.5 & 0.0 & 0.0 & 0.0 & 0.0 & 0.0 & 0.0 \\
\hline 2.8055 & 0.0 & 0.0 & 0.0 & 2.8 & 0.0 & 0.0 & 0.0 & 0.0 & 0.0 & 0.0 \\
\hline 2.8055 & 0.0 & 0.0 & 0.0 & 2.8 & 0.0 & 0.0 & 0.0 & 0.0 & 0.0 & 0.0 \\
\hline 2.8062 & 0.0 & 0.0 & 0.0 & 2.8 & 0.0 & 0.0 & 0.0 & 0.0 & 0.0 & 0.0 \\
\hline \multirow[t]{3}{*}{ Total } & 0.0 & 0.0 & 0.0 & 39.9 & 0.0 & 0.0 & 0.0 & 0.0 & 0.0 & 0.0 \\
\hline & \multicolumn{10}{|c|}{ Total } \\
\hline & 7 & 3 & 8 & 9 & 6 & 7 & 5 & 2 & 4 & 5 \\
\hline 0.7975 & 5.6 & 2.4 & 6.4 & 7.2 & 4.8 & 5.6 & 4.0 & 1.6 & 3.2 & 4.0 \\
\hline 1.2121 & 8.5 & 3.6 & 9.7 & 10.9 & 7.3 & 8.5 & 6.1 & 2.4 & 4.8 & 6.1 \\
\hline 1.3396 & 9.4 & 4.0 & 10.7 & 12.1 & 8.0 & 9.4 & 6.7 & 2.7 & 5.4 & 6.7 \\
\hline 1.4914 & 10.4 & 4.5 & 11.9 & 13.4 & 8.9 & 10.4 & 7.5 & 3.0 & 6.0 & 7.5 \\
\hline 1.5911 & 11.1 & 4.8 & 12.7 & 14.3 & 9.5 & 11.1 & 8.0 & 3.2 & 6.4 & 8.0 \\
\hline 1.7188 & 12.0 & 5.2 & 13.8 & 15.5 & 10.3 & 12.0 & 8.6 & 3.4 & 6.9 & 8.6 \\
\hline 1.8458 & 12.9 & 5.5 & 14.8 & 16.6 & 11.1 & 12.9 & 9.2 & 3.7 & 7.4 & 9.2 \\
\hline 1.8458 & 12.9 & 5.5 & 14.8 & 16.6 & 11.1 & 12.9 & 9.2 & 3.7 & 7.4 & 9.2 \\
\hline 1.8458 & 12.9 & 5.5 & 14.8 & 16.6 & 11.1 & 12.9 & 9.2 & 3.7 & 7.4 & 9.2 \\
\hline 1.8976 & 13.3 & 5.7 & 15.2 & 17.1 & 11.4 & 13.3 & 9.5 & 3.8 & 7.6 & 9.5 \\
\hline 1.9563 & 13.7 & 5.9 & 15.7 & 17.6 & 11.7 & 13.7 & 9.8 & 3.9 & 7.8 & 9.8 \\
\hline 2.0239 & 14.2 & 6.1 & 16.2 & 18.2 & 12.1 & 14.2 & 10.1 & 4.0 & 8.1 & 10.1 \\
\hline 2.1038 & 14.7 & 6.3 & 16.8 & 18.9 & 12.6 & 14.7 & 10.5 & 4.2 & 8.4 & 10.5 \\
\hline 2.3270 & 16.3 & 7.0 & 18.6 & 20.9 & 14.0 & 16.3 & 11.6 & 4.7 & 9.3 & 11.6 \\
\hline 2.5038 & 17.5 & 7.5 & 20.0 & 22.5 & 15.0 & 17.5 & 12.5 & 5.0 & 10.0 & 12.5 \\
\hline 2.5038 & 17.5 & 7.5 & 20.0 & 22.5 & 15.0 & 17.5 & 12.5 & 5.0 & 10.0 & 12.5 \\
\hline 2.5038 & 17.5 & 7.5 & 20.0 & 22.5 & 15.0 & 17.5 & 12.5 & 5.0 & 10.0 & 12.5 \\
\hline 2.8055 & 19.6 & 8.4 & 22.4 & 25.2 & 16.8 & 19.6 & 14.0 & 5.6 & 11.2 & 14.0 \\
\hline 2.8055 & 19.6 & 8.4 & 22.4 & 25.2 & 16.8 & 19.6 & 14.0 & 5.6 & 11.2 & 14.0 \\
\hline 2.8062 & 19.6 & 8.4 & 22.4 & 25.3 & 16.8 & 19.6 & 14.0 & 5.6 & 11.2 & 14.0 \\
\hline Total & 279.5 & 119.8 & 319.4 & 359.3 & 239.5 & 279.5 & 199.6 & 79.8 & 159.7 & 199.6 \\
\hline
\end{tabular}

Predictor 


\section{Continued}

\begin{tabular}{ccccccccccc}
\hline Equatorial Guinea & 0.0 & 0.0 & 39.9 & 0.0 & 0.0 & 0.0 & 0.0 & 0.0 & 0.0 & 0.0 \\
Log & 0.0 & 1.0 & 2.0 & 3.0 & 4.0 & 5.0 & 6.0 & 7.0 & 8.0 & 9.0 \\
Ghana & 119.8 & 39.9 & 279.5 & 119.8 & 0.0 & 79.8 & 79.8 & 39.9 & 159.7 & 79.8 \\
Log & 2.1 & 1.6 & 2.4 & 2.1 & 0.0 & 1.9 & 1.9 & 1.6 & 2.2 & 1.9 \\
Guinea & 159.7 & 79.8 & 0.0 & 199.6 & 239.5 & 199.6 & 119.8 & 39.9 & 159.7 & 119.8 \\
Log & 2.2 & 1.9 & 0.0 & 2.3 & 2.4 & 2.3 & 2.1 & 1.6 & 2.2 & 2.1 \\
Guinea Bissau & 0.0 & 0.0 & 0.0 & 39.9 & 0.0 & 0.0 & 0.0 & 0.0 & 0.0 & 0.0 \\
Log & 0.0 & 0.0 & 0.0 & 1.6 & 0.0 & 0.0 & 0.0 & 0.0 & 0.0 & 0.0 \\
Total & 279.5 & 119.8 & 319.4 & 359.3 & 239.5 & 279.5 & 199.6 & 79.8 & 159.7 & 199.6 \\
Log & 2.4 & 2.1 & 2.5 & 2.6 & 2.4 & 2.4 & 2.3 & 1.9 & 2.2 & 2.3 \\
Sum of Logs & 106.5 & & & & & & & & & \\
Ln (Sum of Logs) & 4.66796 & & & & & & & & &
\end{tabular}

Table 7. Forecasted piracy attacks on gulf of Guinea (Source: Author's compilation from [18] [19]).

\begin{tabular}{lcccccc}
\hline & Equatorial Guinea & Ghana & Guinea & Guinea Bissau & Total & Average \\
\hline 2016 & 4 & 6 & 7 & 4 & 9 & 6 \\
2017 & 9 & 11 & 12 & 9 & 14 & 11 \\
2018 & 14 & 16 & 17 & 14 & 19 & 16 \\
2019 & 19 & 21 & 22 & 19 & 24 & 21 \\
2020 & 24 & 26 & 27 & 24 & 29 & 26 \\
2021 & 29 & 31 & 32 & 29 & 34 & 31 \\
2022 & 34 & 36 & 37 & 34 & 39 & 36 \\
2023 & 39 & 41 & 42 & 39 & 44 & 41 \\
2024 & 44 & 46 & 47 & 44 & 49 & 46 \\
2025 & 49 & 51 & 52 & 49 & 54 & 51 \\
2026 & 54 & 56 & 57 & 54 & 59 & 56 \\
2027 & 59 & 61 & 62 & 59 & 64 & 61 \\
2028 & 64 & 66 & 67 & 64 & 69 & 66 \\
2029 & 69 & 71 & 72 & 69 & 74 & 71 \\
2030 & 74 & 76 & 77 & 74 & 79 & 76 \\
\hline
\end{tabular}

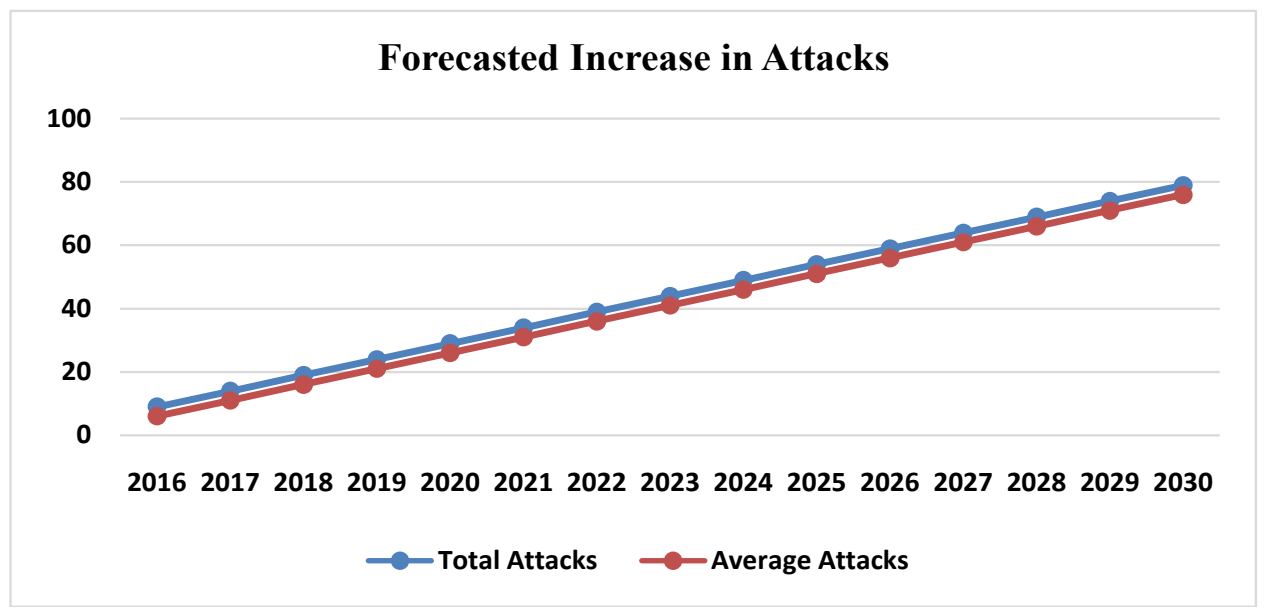

Figure 4. Projected increase in piracy attacks (Source: Author's compilation from [18] [19]). 


\section{Series Hazard Modelling for Maritime Transport Risk Analysis}

\subsection{Background}

According to [27], the hazard of global hijacking by aeroplanes and maritime piracy has been assessed through series hazard modelling for the South China Sea and Malacca Strait. Within the United Kingdom, series hazard modelling has been used to examine republican terrorist attacks. According to [28], series hazard modelling has been used to assess global terrorist attacks in Justice Commandos for the Armenian Genocide (JCAG) and Armenian Secret Army for the Liberation of Armenia (ASALA). Other researchers, such as [29] and [30], from Hamas, Fatah and the Palestinian territories and the Palestinian Islamic Jihad in Israel used series hazard modelling to assess frequent terrorist attacks in Israel. The series hazard modelling method is an important tool for modelling events where events that have taken place will probably be known to those who precipitate future events, because it can directly measure dependence across events. Furthermore, the model can be used to estimate intervention effects across independent events. After getting the appropriate context and data, the hazard model can be used to assess the hidden variation in the duration between events and in the details of each event [28]. The following equation shows the series hazard model:

$$
\lambda_{k}\left(t / X_{k}\right)=\lambda_{0}(t) \exp \left(X_{k} \beta\right)
$$

However, this model is projected using the Cox proportional hazard model. The main difference is that series hazard model is estimated across failures whilst the Cox proportional hazard model is estimated across subjects. Because the history of previous failures has to be captured in the model, to ensure conditional independence, the preceding equation can be expanded into Equation (4) below; where series of functions of previous failures is represented by $Z$, especially those that are relevant theoretically, to the current analysis (in this case maritime piracy events and effects). The partial likelihood function can then be derived from the series hazard function in the following equation:

$$
\lambda_{k}\left(t / X_{k}\right)=\lambda_{0}(t) \exp \left(X_{k} \beta+Z_{k y}\right)
$$

The purpose of the matrix $Z_{k y}$ is to measure information about the history of failures to account for dependencies across failures. The matrix $X_{k}$ consists of information on the specific failure or event, the policy context, political, social reasons at the time of failure (comprising the strategic profile). It is assumed within this model that 1 ) the unit of observation is each failure, not each time increment, and 2) dummy variables may be introduced when necessary.

\subsection{Application of the Data}

For this study, failures were originally found in Table 5 [24] for the ROC curve. Using Equation (4), $\lambda_{0}(t)$ represents FPR, whereas $X$ are the total number of attempts (FPR and TPR) and is also found in Table 5 [24]. In the following ta- 
ble, the log of these values is being considered. Moreover, $\beta$ will be considered AUC in Table 5 [24]. $Z_{k y}$ will be p-Pred found in Table 5 [24]. Once the formula is calculated for the entire dataset, the logs will be found and summed. Following the determination of this summation, the forecast will be determined based on the sum of the logs and conducted as done with the preceding model. The following Table 8 presents the results of the equation for the dataset.

Table 8. Series hazard model (Source: Adapted from [28] [29]).

\begin{tabular}{|c|c|c|c|c|c|}
\hline$\lambda_{0}(t)=$ Failures & Success & $X=$ Column $1+2$ & $\beta$ & $Z_{k y}$ & \\
\hline 1.0000 & 1.0000 & 2.0000 & 0.0882 & 0.0000 & 1.0000 \\
\hline 0.9118 & 1.0000 & 1.9118 & 0.0611 & 0.0003 & 0.9943 \\
\hline 0.8507 & 1.0000 & 1.8507 & 0.0087 & 0.0009 & 0.9984 \\
\hline 0.8420 & 0.9949 & 1.8369 & 0.0060 & 0.0155 & 0.9963 \\
\hline 0.8360 & 0.9949 & 1.8309 & 0.0173 & 0.0239 & 0.9926 \\
\hline 0.8186 & 0.9949 & 1.8135 & 0.0051 & 0.0255 & 0.9939 \\
\hline 0.8135 & 0.9872 & 1.8007 & 0.0033 & 0.0325 & 0.9926 \\
\hline 0.8101 & 0.9821 & 1.7922 & 0.0059 & 0.0362 & 0.9912 \\
\hline 0.8041 & 0.9692 & 1.7733 & 0.0017 & 0.0362 & 0.9918 \\
\hline 0.8023 & 0.9692 & 1.7715 & 0.0014 & 0.0372 & 0.9915 \\
\hline 0.8009 & 0.9692 & 1.7701 & 0.0070 & 0.0427 & 0.9890 \\
\hline 0.7936 & 0.9462 & 1.7398 & 0.0015 & 0.0430 & 0.9898 \\
\hline 0.7920 & 0.9462 & 1.7382 & 0.0017 & 0.0435 & 0.9895 \\
\hline 0.7903 & 0.9436 & 1.7339 & 0.0074 & 0.0486 & 0.9869 \\
\hline 0.7825 & 0.9436 & 1.7261 & 0.0010 & 0.0490 & 0.9878 \\
\hline 0.7814 & 0.9436 & 1.7250 & 0.0017 & 0.0498 & 0.9874 \\
\hline 0.7796 & 0.9333 & 1.7129 & 0.0023 & 0.0504 & 0.9870 \\
\hline 0.7771 & 0.9308 & 1.7079 & 0.0020 & 0.0505 & 0.9868 \\
\hline 0.7750 & 0.9256 & 1.7006 & 0.0007 & 0.0509 & 0.9869 \\
\hline 0.7743 & 0.9256 & 1.6999 & 0.0003 & 0.0518 & 0.9868 \\
\hline 0.7740 & 0.9256 & 1.6996 & 0.0002 & 0.0519 & 0.9867 \\
\hline 0.7738 & 0.9256 & 1.6994 & 0.0002 & 0.0526 & 0.9866 \\
\hline 0.7736 & 0.9256 & 1.6992 & 0.0002 & 0.0526 & 0.9865 \\
\hline 0.7734 & 0.9256 & 1.6990 & 0.0002 & 0.0526 & 0.9865 \\
\hline 0.7733 & 0.9256 & 1.6989 & 0.0010 & 0.0526 & 0.9863 \\
\hline 0.7722 & 0.9256 & 1.6978 & 0.0007 & 0.0541 & 0.9859 \\
\hline 0.7715 & 0.9256 & 1.6971 & 0.0002 & 0.0549 & 0.9858 \\
\hline 0.7713 & 0.9256 & 1.6969 & 0.0002 & 0.0550 & 0.9858 \\
\hline 0.7711 & 0.9256 & 1.6967 & 0.0002 & 0.0550 & 0.9858 \\
\hline 0.7709 & 0.9256 & 1.6965 & 0.0002 & 0.0550 & 0.9857 \\
\hline 0.7708 & 0.9256 & 1.6964 & 0.0002 & 0.0550 & 0.9857 \\
\hline 0.7706 & 0.9256 & 1.6962 & 0.0002 & 0.0550 & 0.9857 \\
\hline 0.7704 & 0.9256 & 1.6960 & 0.0002 & 0.0582 & 0.9849 \\
\hline 0.7702 & 0.9256 & 1.6958 & 0.0002 & 0.0582 & 0.9849 \\
\hline 0.7701 & 0.9256 & 1.6957 & 0.0002 & 0.0582 & 0.9849 \\
\hline 0.7699 & 0.9256 & 1.6955 & 0.0043 & 0.0582 & 0.9838 \\
\hline 0.7653 & 0.9231 & 1.6884 & 0.0005 & 0.0588 & 0.9843 \\
\hline
\end{tabular}


N. R. L. Ofosu-Boateng

\section{Continued}

\begin{tabular}{|c|c|c|c|c|c|}
\hline 0.7647 & 0.9231 & 1.6878 & 0.0016 & 0.0590 & 0.9839 \\
\hline 0.7630 & 0.9205 & 1.6835 & 0.0049 & 0.0592 & 0.9828 \\
\hline 0.7577 & 0.9205 & 1.6782 & 0.0018 & 0.0606 & 0.9828 \\
\hline 0.7557 & 0.9179 & 1.6736 & 0.0111 & 0.0607 & 0.9801 \\
\hline 0.7437 & 0.9179 & 1.6616 & 0.0002 & 0.0615 & 0.9819 \\
\hline 0.7435 & 0.9179 & 1.6614 & 0.0002 & 0.0627 & 0.9815 \\
\hline 0.7433 & 0.9179 & 1.6612 & 0.0002 & 0.0627 & 0.9815 \\
\hline 0.7431 & 0.9179 & 1.6610 & 0.0010 & 0.0627 & 0.9813 \\
\hline 0.7421 & 0.9128 & 1.6549 & 0.0036 & 0.0635 & 0.9802 \\
\hline 0.7382 & 0.9103 & 1.6485 & 0.0390 & 0.0650 & 0.9689 \\
\hline 0.6953 & 0.8744 & 1.5697 & 0.0033 & 0.0650 & 0.9755 \\
\hline 0.6916 & 0.8744 & 1.5660 & 0.0014 & 0.0663 & 0.9753 \\
\hline 0.6900 & 0.8744 & 1.5644 & 0.0019 & 0.0663 & 0.9750 \\
\hline 0.6879 & 0.8744 & 1.5623 & 0.0003 & 0.0672 & 0.9751 \\
\hline 0.6875 & 0.8744 & 1.5619 & 0.0014 & 0.0677 & 0.9744 \\
\hline 0.6859 & 0.8744 & 1.5603 & 0.0037 & 0.0693 & 0.9729 \\
\hline 0.6817 & 0.8718 & 1.5535 & 0.0006 & 0.0698 & 0.9734 \\
\hline 0.6810 & 0.8718 & 1.5528 & 0.0053 & 0.0722 & 0.9707 \\
\hline 0.6749 & 0.8590 & 1.5339 & 0.4764 & 0.0732 & 0.8057 \\
\hline 0.1203 & 0.2205 & 0.3408 & 0.0010 & 0.0735 & 0.8540 \\
\hline 0.1159 & 0.2154 & 0.3313 & 0.0069 & 0.0777 & 0.8333 \\
\hline 0.0840 & 0.1872 & 0.2712 & 0.0008 & 0.0784 & 0.8219 \\
\hline 0.0799 & 0.1872 & 0.2671 & 0.0045 & 0.0862 & 0.7952 \\
\hline 0.0556 & 0.1538 & 0.2094 & 0.0014 & 0.0870 & 0.7746 \\
\hline 0.0462 & 0.1256 & 0.1718 & 0.0015 & 0.1134 & 0.7024 \\
\hline 0.0340 & 0.1026 & 0.1366 & 0.0018 & 0.1196 & 0.6633 \\
\hline 0.0168 & 0.0692 & 0.0860 & 0.0012 & 0.1300 & 0.5850 \\
\hline & \multicolumn{5}{|c|}{ Predictor } \\
\hline \multirow[t]{13}{*}{ Log (Column 6) } & 0.0000 & -0.0057 & -0.0062 & -0.0075 & -0.0120 \\
\hline & -0.0025 & -0.0053 & -0.0062 & -0.0087 & -0.0117 \\
\hline & -0.0007 & -0.0055 & -0.0062 & -0.0079 & -0.0129 \\
\hline & -0.0016 & -0.0057 & -0.0062 & -0.0081 & -0.0939 \\
\hline & -0.0032 & -0.0057 & -0.0062 & -0.0081 & -0.0685 \\
\hline & -0.0027 & -0.0057 & -0.0062 & -0.0082 & -0.0792 \\
\hline & -0.0032 & -0.0058 & -0.0066 & -0.0087 & -0.0852 \\
\hline & -0.0039 & -0.0058 & -0.0066 & -0.0137 & -0.0995 \\
\hline & -0.0036 & -0.0059 & -0.0066 & -0.0108 & -0.1109 \\
\hline & -0.0037 & -0.0059 & -0.0071 & -0.0108 & -0.1534 \\
\hline & -0.0048 & -0.0059 & -0.0069 & -0.0110 & -0.1783 \\
\hline & -0.0045 & -0.0060 & -0.0071 & -0.0110 & -0.2328 \\
\hline & -0.0046 & -0.0062 & -0.0075 & -0.0112 & \\
\hline Sum of Logs & -1.4639 & & & & \\
\hline
\end{tabular}


The formula results in the following Figure 5.

Based on these results, it is estimated that the prediction rate is accurate for determining the success of piracy attacks. The forecast predictor is shown in the following Table 9 and is based on the sum of the logs (shown in Table 8 [28] [29]) to the power of 1 through 5 (finding the absolute value of the total for each country).

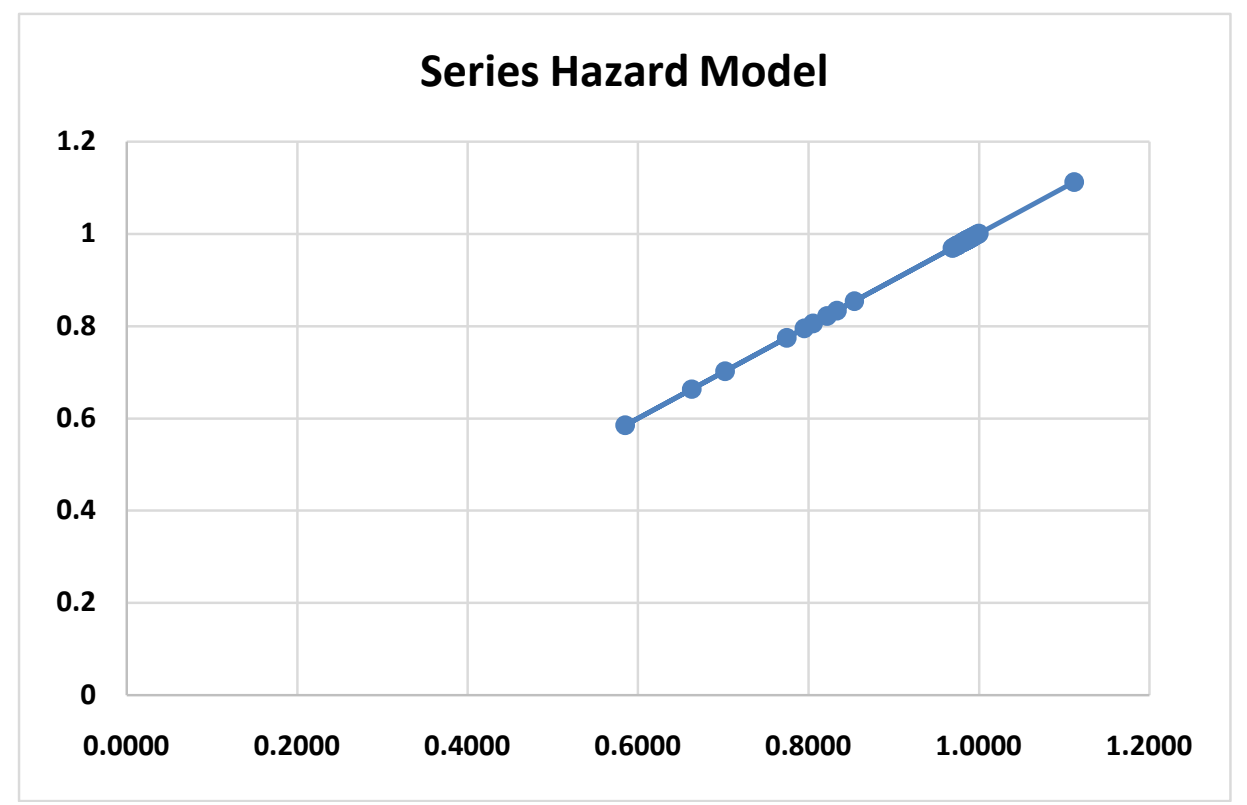

Figure 5. Series hazard modeling (Source: Adapted from [28]).

Table 9. Forecast based on series hazard modeling (Source: Author' compilation [18] [19]).

\begin{tabular}{ccccccccccc}
\hline Location & $\mathbf{0 6}$ & $\mathbf{0 7}$ & $\mathbf{0 8}$ & $\mathbf{0 9}$ & $\mathbf{1 0}$ & $\mathbf{1 1}$ & $\mathbf{1 2}$ & $\mathbf{1 3}$ & $\mathbf{1 4}$ & $\mathbf{1 5}$ \\
\hline Equatorial Guinea & 0 & 0 & 1 & 0 & 0 & 0 & 0 & 0 & 0 & 0 \\
Ghana & 3 & 1 & 7 & 3 & 0 & 2 & 2 & 1 & 4 & 2 \\
Guinea & 4 & 2 & 0 & 5 & 6 & 5 & 3 & 1 & 0 & 3 \\
Guinea Bissau & 0 & 0 & 0 & 1 & 0 & 0 & 0 & 0 & 0 & 0 \\
Total & 7 & 3 & 8 & 9 & 6 & 7 & 5 & 2 & 4 & 5 \\
\hline & & & & Equatorial Guinea & & & \\
\hline & $\mathbf{0}$ & $\mathbf{0}$ & $\mathbf{1}$ & $\mathbf{0}$ & $\mathbf{0}$ & $\mathbf{0}$ & $\mathbf{0}$ & $\mathbf{0}$ & $\mathbf{0}$ & $\mathbf{0}$ \\
\hline-1.4639 & 0.0 & 0.0 & -1.5 & 0.0 & 0.0 & 0.0 & 0.0 & 0.0 & 0.0 & 0.0 \\
2.1431 & 0.0 & 0.0 & 2.1 & 0.0 & 0.0 & 0.0 & 0.0 & 0.0 & 0.0 & 0.0 \\
-3.1373 & 0.0 & 0.0 & -3.1 & 0.0 & 0.0 & 0.0 & 0.0 & 0.0 & 0.0 & 0.0 \\
4.5927 & 0.0 & 0.0 & 4.6 & 0.0 & 0.0 & 0.0 & 0.0 & 0.0 & 0.0 & 0.0 \\
-6.7234 & 0.0 & 0.0 & -6.7 & 0.0 & 0.0 & 0.0 & 0.0 & 0.0 & 0.0 & 0.0 \\
Total & $\mathbf{0 . 0}$ & $\mathbf{0 . 0}$ & $\mathbf{4 . 6}$ & $\mathbf{0 . 0}$ & $\mathbf{0 . 0}$ & $\mathbf{0 . 0}$ & $\mathbf{0 . 0}$ & $\mathbf{0 . 0}$ & $\mathbf{0 . 0}$ & $\mathbf{0 . 0}$ \\
\hline & & & & & $\mathbf{G h a n a}$ & & & & \\
\hline & $\mathbf{3}$ & $\mathbf{1}$ & $\mathbf{7}$ & $\mathbf{3}$ & $\mathbf{0}$ & $\mathbf{2}$ & $\mathbf{2}$ & $\mathbf{1}$ & $\mathbf{4}$ & $\mathbf{2}$ \\
\hline-1.4639 & -4.4 & -1.5 & -10.2 & -4.4 & 0.0 & -2.9 & -2.9 & -1.5 & -5.9 & -2.9 \\
\hline
\end{tabular}




\section{Continued}

\begin{tabular}{|c|c|c|c|c|c|c|c|c|c|c|}
\hline 2.1431 & 6.4 & 2.1 & 15.0 & 6.4 & 0.0 & 4.3 & 4.3 & 2.1 & 8.6 & 4.3 \\
\hline-3.1373 & -9.4 & -3.1 & -22.0 & -9.4 & 0.0 & -6.3 & -6.3 & -3.1 & -12.5 & -6.3 \\
\hline 4.5927 & 13.8 & 4.6 & 32.1 & 13.8 & 0.0 & 9.2 & 9.2 & 4.6 & 18.4 & 9.2 \\
\hline-6.7234 & -20.2 & -6.7 & -47.1 & -20.2 & 0.0 & -13.4 & -13.4 & -6.7 & -26.9 & -13.4 \\
\hline \multirow[t]{3}{*}{ Total } & 13.8 & 4.6 & 32.1 & 13.8 & 0.0 & 9.2 & 9.2 & 4.6 & 18.4 & 9.2 \\
\hline & \multicolumn{10}{|c|}{ Guinea } \\
\hline & 4 & 2 & 0 & 5 & 6 & 5 & 3 & 1 & 0 & 3 \\
\hline-1.4639 & -5.9 & -2.9 & 0.0 & -7.3 & -8.8 & -7.3 & -4.4 & -1.5 & -5.9 & -4.4 \\
\hline 2.1431 & 8.6 & 4.3 & 0.0 & 10.7 & 12.9 & 10.7 & 6.4 & 2.1 & 8.6 & 6.4 \\
\hline-3.1373 & -12.5 & -6.3 & 0.0 & -15.7 & $7-18.8$ & -15.7 & -9.4 & -3.1 & -12.5 & -9.4 \\
\hline 4.5927 & 18.4 & 9.2 & 0.0 & 23.0 & 27.6 & 23.0 & 13.8 & 4.6 & 18.4 & 13.8 \\
\hline-6.7234 & -26.9 & -13.4 & 0.0 & -33.6 & -40.3 & -33.6 & -20.2 & -6.7 & -26.9 & -20.2 \\
\hline \multirow[t]{3}{*}{ Total } & 18.4 & 9.2 & 0.0 & 22.9 & 27.5 & 22.9 & 13.8 & 4.6 & 18.4 & 13.8 \\
\hline & \multicolumn{10}{|c|}{ Guinea Bissau } \\
\hline & 0 & 0 & 0 & 1 & 0 & 0 & 0 & 0 & 0 & 0 \\
\hline-1.4639 & 0.0 & 0.0 & 0.0 & -1.5 & 0.0 & 0.0 & 0.0 & 0.0 & 0.0 & 0.0 \\
\hline 2.1431 & 0.0 & 0.0 & 0.0 & 2.1 & 0.0 & 0.0 & 0.0 & 0.0 & 0.0 & 0.0 \\
\hline-3.1373 & 0.0 & 0.0 & 0.0 & -3.1 & 0.0 & 0.0 & 0.0 & 0.0 & 0.0 & 0.0 \\
\hline 4.5927 & 0.0 & 0.0 & 0.0 & 4.6 & 0.0 & 0.0 & 0.0 & 0.0 & 0.0 & 0.0 \\
\hline-6.7234 & 0.0 & 0.0 & 0.0 & -6.7 & 0.0 & 0.0 & 0.0 & 0.0 & 0.0 & 0.0 \\
\hline \multirow[t]{3}{*}{ Total } & 0.0 & 0.0 & 0.0 & 4.6 & 0.0 & 0.0 & 0.0 & 0.0 & 0.0 & 0.0 \\
\hline & \multicolumn{10}{|c|}{ Total } \\
\hline & 7 & 3 & 8 & 9 & 6 & 7 & 5 & 2 & 4 & 5 \\
\hline-1.4639 & -10.2 & -4.4 & -11.7 & -13.2 & -8.8 & -10.2 & -7.3 & -2.9 & -5.9 & -7.3 \\
\hline 2.1431 & 15.0 & 6.4 & 17.1 & 19.3 & 12.9 & 15.0 & 10.7 & 4.3 & 8.6 & 10.7 \\
\hline-3.1373 & -22.0 & -9.4 & -25.1 & -28.2 & -18.8 & -22.0 & -15.7 & -6.3 & -12.5 & -15.7 \\
\hline 4.5927 & 32.1 & 13.8 & 36.7 & 41.3 & 27.6 & 32.1 & 23.0 & 9.2 & 18.4 & 23.0 \\
\hline-6.7234 & -47.1 & -20.2 & -53.8 & -60.5 & -40.3 & -47.1 & -33.6 & -13.4 & -26.9 & -33.6 \\
\hline \multirow[t]{2}{*}{ Total } & 32.1 & 13.8 & 36.7 & 41.3 & 27.5 & 32.1 & 22.9 & 9.2 & 18.4 & 22.9 \\
\hline & \multicolumn{10}{|c|}{ Predictor } \\
\hline Equatorial Guinea & 0.0 & 0.0 & 4.6 & 0.0 & 0.0 & 0.0 & 0.0 & 0.0 & 0.0 & 0.0 \\
\hline $\log$ & 0.0 & 0.0 & 0.7 & 0.0 & 0.0 & 0.0 & 0.0 & 0.0 & 0.0 & 0.0 \\
\hline Ghana & 13.8 & 4.6 & 32.1 & 13.8 & 0.0 & 9.2 & 9.2 & 4.6 & 18.4 & 9.2 \\
\hline $\log$ & 1.1 & 0.7 & 1.5 & 1.1 & 0.0 & 1.0 & 1.0 & 0.7 & 1.3 & 1.0 \\
\hline Guinea & 18.4 & 9.2 & 0.0 & 22.9 & 27.5 & 22.9 & 13.8 & 4.6 & 18.4 & 13.8 \\
\hline $\log$ & 1.3 & 1.0 & 0.0 & 1.4 & 1.4 & 1.4 & 1.1 & 0.7 & 1.3 & 1.1 \\
\hline Guinea Bissau & 0.0 & 0.0 & 0.0 & 4.6 & 0.0 & 0.0 & 0.0 & 0.0 & 0.0 & 0.0 \\
\hline $\log$ & 0.0 & 0.0 & 0.0 & 0.7 & 0.0 & 0.0 & 0.0 & 0.0 & 0.0 & 0.0 \\
\hline Total & 32.1 & 13.8 & 36.7 & 41.3 & 27.5 & 32.1 & 22.9 & 9.2 & 18.4 & 22.9 \\
\hline $\log$ & 1.5 & 1.1 & 1.6 & 1.6 & 1.4 & 1.5 & 1.4 & 1.0 & 1.3 & 1.4 \\
\hline Sum of Logs & \multicolumn{2}{|c|}{34.9} & & & & & & & & \\
\hline Ln (Sum of Logs) & \multicolumn{2}{|c|}{3.55234} & & & & & & & & \\
\hline
\end{tabular}


Based on this information, the increase is by 4 annually, as shown in Table 10 and graph Figure 6.

\section{Bayesian Networks (BNs) Technique for Maritime Risk Analysis}

\subsection{Background}

[31] [32] [33] [34] and [35] suggest that another risk modelling tool that can be used for maritime transportation analysis is Bayesian Networks, which have a number of favourable features. Through situational factors, Bayesian Networks can allow for the contextualization of specific consequences occurrences, such as

Table 10. Forecasted piracy attacks on gulf of Guinea (Source: Author's compilation [18] [19]).

\begin{tabular}{|c|c|c|c|c|c|c|}
\hline & Equatorial Guinea & Ghana & Guinea & Guinea Bissau & Total & Average \\
\hline 2016 & 4 & 6 & 7 & 4 & 9 & 6 \\
\hline 2017 & 8 & 10 & 11 & 8 & 13 & 10 \\
\hline 2018 & 12 & 14 & 15 & 12 & 17 & 14 \\
\hline 2019 & 16 & 18 & 19 & 16 & 21 & 18 \\
\hline 2020 & 20 & 22 & 23 & 20 & 25 & 22 \\
\hline 2021 & 24 & 26 & 27 & 24 & 29 & 26 \\
\hline 2022 & 28 & 30 & 31 & 28 & 33 & 30 \\
\hline 2023 & 32 & 34 & 35 & 32 & 37 & 34 \\
\hline 2024 & 36 & 38 & 39 & 36 & 41 & 38 \\
\hline 2025 & 40 & 42 & 43 & 40 & 45 & 42 \\
\hline 2026 & 44 & 46 & 47 & 44 & 49 & 46 \\
\hline 2027 & 48 & 50 & 51 & 48 & 53 & 50 \\
\hline 2028 & 52 & 54 & 55 & 52 & 57 & 54 \\
\hline 2029 & 56 & 58 & 59 & 56 & 61 & 58 \\
\hline 2030 & 60 & 62 & 63 & 60 & 65 & 62 \\
\hline
\end{tabular}

\section{Forecasted Increase in Attacks}

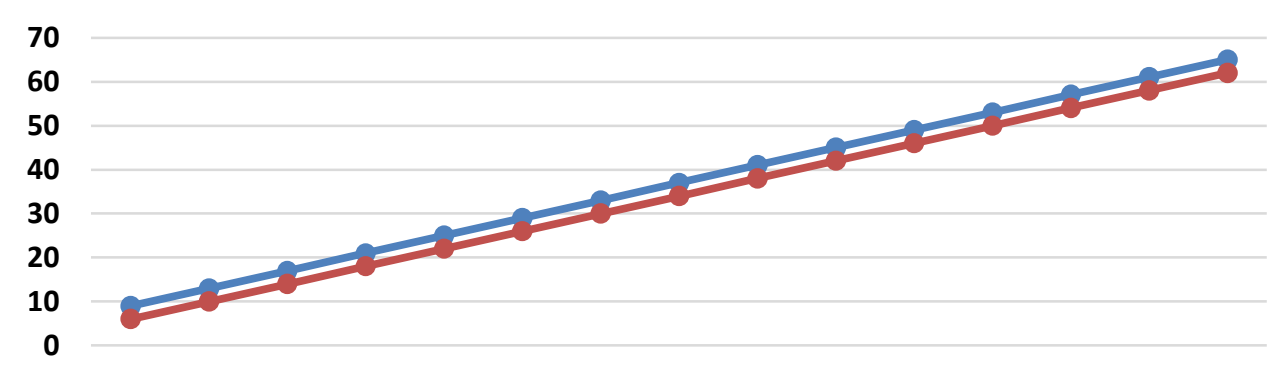

201620172018201920202021202220232024202520262027202820292030

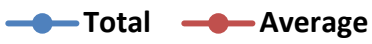

Figure 6. Projected increase in piracy attacks (Source: Author's compilation from [18] [19]). 
piracy and its related effects, represented by observable aspects. In addition, by providing a means for performing sensitivity analysis, BNs allows for the integration of different types of evidence through various types of probabilities.

[36] suggests that BNs constitute probabilistic graphical models, defined as a pair $\Delta=\{G(V, A), P\}$, where $G(V, A)$ is the graphical constituent and $P$ the probabilistic constituent of the model. Where the arcs $(A)$ represent the conditional (in) dependence relationships between these, the nodes represent the variables $V=\{V 1, \cdots, V n\}, G(V, A)$ becomes a directed acyclic graph (DAG), shown below:

$$
P(V)=\prod_{i=1}^{n}\left(V_{i} \mid \operatorname{Pa}\left(V_{i}\right)\right)
$$

In this formula, $P\left(V_{i} \mid P a\left(V_{i}\right)\right)$ for each variable $V i, i=1, \cdots, n$ in the network; $P$ is a set of conditional probability tables; $P a(V i)=\{Y \in V \mid(Y, V i) \in A\}$; and $\mathrm{Pa}(\mathrm{Vi})$ represents the set of parents of $V i$ in $G$. Therefore, $P=\{P(V i \mid P a(V i)), i=1, \cdots, n\}$. A BN encodes a factorization of the joint probability distribution (JDP) over all variables in $V$. A sensitivity analysis can then be performed for further information [36].

\subsection{Application to the Data}

Within this study, again, probability can be found in both Table 5 [24] and Table 8 [28] [29]. For this scenario, the probability is considered p-Pred and is multiplied by the variables found in the appendix. For each row, the sum will be determined then $\log (\mathrm{sum})$ again. If this value is between -0.15 to 0.20 , this value is reliable and the predictor can be used to determine the forecast predictor. If the value is outside of the range, the log will be found of the $\log (\mathrm{sum})$, which will be added to the p-Pred used for the forecast model. The following Table 11 shows the initial results.

Based on these results, it is estimated that the prediction rate will need to have 0.33052 added for determining the success of piracy attacks. The forecast predictor is shown in the following Table 12.

Based on this information, the increase is by 3 annually, as shown in Table 13 and Figure 7.

\section{Conclusion}

The chapter considers three separate models: Ordinal Logistic Regression; Series Hazard Modelling for maritime transport risk analysis; and Bayesian Networks (BN's) technique for maritime risk analysis to analyse one set of data. It is noted that all of the models used yielded similar forecasted results. Moreover, all models showed that the Gulf of Guinea is expected to have a significant increase in piracy through 2030. As explored in the introductory chapter, this could be due to increases in oil piracy. Therefore, it will be important for ship owners and crews to be vigilant while on route to destinations in these areas or through these waters in order to prevent these crimes from occurring. The long coastline and territorial seas bordering the Gulf of Guinea will have to be effectively monitored 
Table 11. Bayesian model predictor (Source: Author's compilation with data from [18] [19]).

\begin{tabular}{|c|c|c|c|c|c|c|c|c|c|c|}
\hline \multirow[b]{2}{*}{06} & \multicolumn{9}{|c|}{$20 \mathrm{xx}$} & \multirow[b]{2}{*}{ Sum } \\
\hline & 07 & 08 & 09 & 10 & 11 & 12 & 13 & 14 & 15 & \\
\hline 0.0 & 0.0 & 0.0 & 0.0 & 0.0 & 0.0 & 0.0 & 0.0 & 0.0 & 0.0 & 0.2 \\
\hline 0.0 & 0.0 & 0.0 & 0.0 & 0.0 & 0.0 & 0.0 & 0.0 & 0.0 & 0.0 & 0.0 \\
\hline 0.2 & 0.1 & 0.2 & 0.2 & 0.3 & 0.2 & 0.2 & 0.1 & 0.4 & 0.2 & 2.1 \\
\hline 0.0 & 0.0 & 0.0 & 0.0 & 0.0 & 0.0 & 0.0 & 0.0 & 0.0 & 0.0 & 0.1 \\
\hline 0.2 & 0.2 & 0.2 & 0.0 & 0.1 & 0.1 & 0.1 & 0.1 & 0.2 & 0.3 & 1.4 \\
\hline 0.2 & 0.1 & 0.2 & 0.3 & 0.1 & 0.4 & 0.2 & 0.3 & 0.3 & 0.3 & 2.2 \\
\hline 0.0 & 0.1 & 0.0 & 0.1 & 0.1 & 0.0 & 0.0 & 0.0 & 0.1 & 0.0 & 0.4 \\
\hline 0.0 & 0.0 & 0.0 & 0.0 & 0.0 & 0.1 & 0.0 & 0.0 & 0.0 & 0.1 & 0.4 \\
\hline 0.0 & 0.0 & 0.0 & 0.0 & 0.0 & 0.0 & 0.0 & 0.0 & 0.0 & 0.0 & 0.0 \\
\hline 0.0 & 0.0 & 0.0 & 0.0 & 0.0 & 0.0 & 0.0 & 0.0 & 0.0 & 0.0 & 0.0 \\
\hline 0.0 & 0.1 & 0.0 & 0.6 & 1.3 & 0.6 & 0.1 & 0.2 & 0.0 & 0.0 & 2.9 \\
\hline 0.1 & 0.2 & 0.5 & 0.4 & 0.5 & 0.3 & 0.2 & 0.4 & 0.3 & 1.2 & 4.1 \\
\hline 2.3 & 0.7 & 0.6 & 0.9 & 1.1 & 0.5 & 0.5 & 0.6 & 1.0 & 0.5 & 8.7 \\
\hline 0.2 & 0.5 & 0.5 & 0.6 & 0.2 & 0.3 & 0.4 & 0.7 & 0.6 & 0.6 & 4.8 \\
\hline 0.0 & 0.2 & 0.0 & 0.0 & 0.0 & 0.0 & 0.0 & 0.0 & 0.0 & 0.0 & 0.3 \\
\hline 0.1 & 0.0 & 0.0 & 0.0 & 0.0 & 0.0 & 0.0 & 0.0 & 0.0 & 0.0 & 0.1 \\
\hline 0.4 & 0.2 & 0.1 & 0.3 & 0.5 & 0.2 & 0.1 & 0.1 & 0.1 & 0.5 & 2.1 \\
\hline 0.1 & 0.0 & 0.1 & 0.3 & 0.2 & 0.2 & 0.3 & 0.4 & 0.1 & 0.3 & 1.7 \\
\hline 0.0 & 0.0 & 0.0 & 0.2 & 0.1 & 0.2 & 0.1 & 0.0 & 0.0 & 0.0 & 0.4 \\
\hline 0.1 & 0.0 & 0.1 & 0.1 & 0.2 & 0.3 & 0.2 & 0.2 & 0.0 & 0.0 & 1.1 \\
\hline 0.1 & 0.3 & 0.0 & 0.0 & 0.1 & 0.1 & 0.0 & 0.1 & 0.1 & 0.0 & 0.6 \\
\hline 0.0 & 0.1 & 0.1 & 0.2 & 0.3 & 0.1 & 0.1 & 0.0 & 0.0 & 0.1 & 1.0 \\
\hline 0.2 & 0.1 & 0.0 & 0.0 & 0.0 & 0.0 & 0.0 & 0.0 & 0.0 & 0.0 & 0.2 \\
\hline 0.5 & 0.3 & 0.3 & 0.7 & 0.5 & 0.1 & 0.1 & 0.0 & 0.0 & 0.1 & 2.6 \\
\hline 0.0 & 0.1 & 0.0 & 0.0 & 0.0 & 0.0 & 0.0 & 0.0 & 0.0 & 0.0 & 0.1 \\
\hline 0.1 & 0.0 & 0.0 & 0.0 & 0.0 & 0.0 & 0.0 & 0.0 & 0.0 & 0.0 & 0.1 \\
\hline 0.2 & 0.1 & 0.2 & 0.3 & 0.4 & 0.2 & 0.0 & 0.0 & 0.1 & 0.1 & 1.4 \\
\hline 0.2 & 0.1 & 0.1 & 0.0 & 0.0 & 0.1 & 0.0 & 0.0 & 0.1 & 0.0 & 0.5 \\
\hline 0.0 & 0.0 & 0.0 & 0.1 & 0.0 & 1.1 & 0.1 & 0.0 & 0.0 & 0.0 & 1.3 \\
\hline 0.1 & 0.0 & 0.1 & 0.2 & 0.3 & 0.0 & 0.1 & 0.0 & 0.1 & 0.1 & 0.8 \\
\hline 0.0 & 0.0 & 0.1 & 0.0 & 0.1 & 0.2 & 0.1 & 0.0 & 0.1 & 0.1 & 0.6 \\
\hline 0.2 & 0.2 & 0.1 & 0.1 & 0.2 & 0.2 & 0.2 & 0.2 & 0.4 & 0.0 & 1.7 \\
\hline 0.0 & 0.1 & 0.0 & 0.0 & 0.1 & 0.2 & 0.4 & 0.4 & 0.0 & 0.1 & 1.3 \\
\hline 0.0 & 0.0 & 0.1 & 0.0 & 0.0 & 0.0 & 0.0 & 0.0 & 0.0 & 0.0 & 0.1 \\
\hline 0.0 & 0.1 & 0.0 & 0.0 & 0.0 & 0.0 & 0.0 & 0.0 & 0.0 & 0.0 & 0.1 \\
\hline 0.2 & 0.1 & 0.4 & 0.2 & 0.0 & 0.1 & 0.1 & 0.1 & 0.2 & 0.1 & 1.5 \\
\hline 0.2 & 0.1 & 0.0 & 0.3 & 0.4 & 0.3 & 0.2 & 0.1 & 0.0 & 0.2 & 1.7 \\
\hline 0.0 & 0.0 & 0.0 & 0.1 & 0.0 & 0.0 & 0.0 & 0.0 & 0.0 & 0.0 & 0.1 \\
\hline
\end{tabular}




\section{Continued}

\begin{tabular}{|c|c|c|c|c|c|c|c|c|c|c|}
\hline 0.6 & 0.8 & 5.6 & 7.1 & 3.2 & 2.2 & 0.8 & 0.4 & 0.2 & 0.0 & 20.9 \\
\hline 0.1 & 0.0 & 0.2 & 0.1 & 0.2 & 0.1 & 0.3 & 0.2 & 0.2 & 0.1 & 1.5 \\
\hline 0.0 & 0.2 & 0.1 & 0.1 & 0.0 & 0.1 & 0.1 & 0.1 & 0.0 & 0.1 & 0.7 \\
\hline 0.0 & 0.1 & 0.1 & 0.0 & 0.1 & 0.0 & 0.0 & 0.0 & 0.1 & 0.1 & 0.4 \\
\hline 0.0 & 0.1 & 0.0 & 0.0 & 0.0 & 0.0 & 0.0 & 0.0 & 0.0 & 0.0 & 0.1 \\
\hline 0.1 & 0.0 & 0.0 & 0.0 & 0.0 & 0.0 & 0.0 & 0.1 & 0.0 & 0.0 & 0.1 \\
\hline 0.0 & 0.1 & 0.1 & 0.0 & 0.0 & 0.0 & 0.0 & 0.1 & 0.1 & 0.0 & 0.3 \\
\hline 0.0 & 0.2 & 0.1 & 0.0 & 0.0 & 0.0 & 0.1 & 0.1 & 0.1 & 0.1 & 0.7 \\
\hline 0.8 & 2.7 & 2.6 & 1.9 & 1.2 & 0.7 & 1.8 & 2.0 & 1.2 & 0.9 & 15.7 \\
\hline 0.0 & 0.0 & 0.0 & 1.0 & 1.7 & 2.6 & 0.9 & 0.1 & 0.3 & 0.0 & 6.5 \\
\hline 0.1 & 0.1 & 0.0 & 0.0 & 0.0 & 0.1 & 0.1 & 0.1 & 0.1 & 0.0 & 0.6 \\
\hline 0.7 & 2.1 & 1.3 & 5.4 & 9.3 & 10.8 & 3.3 & 0.5 & 0.2 & 0.0 & 33.5 \\
\hline 0.6 & 0.7 & 0.9 & 0.3 & 0.1 & 0.0 & 0.1 & 0.1 & 0.1 & 0.0 & 3.0 \\
\hline 0.1 & 0.0 & 0.1 & 0.1 & 0.0 & 0.4 & 1.0 & 0.5 & 0.1 & 0.0 & 2.4 \\
\hline 0.1 & 0.3 & 0.0 & 0.1 & 0.1 & 0.0 & 0.0 & 0.0 & 0.0 & 0.0 & 0.6 \\
\hline 0.1 & 0.0 & 0.0 & 0.0 & 0.0 & 0.0 & 0.0 & 0.0 & 0.0 & 0.0 & 0.0 \\
\hline 0.0 & 0.0 & 0.0 & 0.0 & 0.1 & 0.0 & 0.0 & 0.0 & 0.0 & 0.0 & 0.0 \\
\hline 0.0 & 0.0 & 0.0 & 0.1 & 0.0 & 0.0 & 0.0 & 0.0 & 0.0 & 0.0 & 0.0 \\
\hline 0.0 & 0.0 & 0.0 & 0.0 & 0.1 & 0.0 & 0.0 & 0.0 & 0.0 & 0.0 & 0.0 \\
\hline 0.0 & 0.0 & 0.0 & 0.0 & 0.1 & 0.0 & 0.0 & 0.0 & 0.0 & 0.0 & 0.0 \\
\hline 0.2 & 0.2 & 0.2 & 0.0 & 0.0 & 0.0 & 0.0 & 0.0 & 0.0 & 0.0 & 0.0 \\
\hline 0.2 & 0.2 & 0.2 & 0.0 & 0.0 & 0.2 & 0.0 & 0.0 & 0.0 & 0.0 & 0.0 \\
\hline 0.0 & 0.3 & 0.3 & 0.0 & 0.5 & 0.0 & 0.1 & 0.0 & 0.0 & 0.2 & 0.0 \\
\hline 0.1 & 0.0 & 0.0 & 0.0 & 0.0 & 0.0 & 0.0 & 0.0 & 0.0 & 0.0 & 0.0 \\
\hline 0.0 & 0.0 & 0.0 & 0.1 & 0.0 & 0.0 & 0.0 & 0.0 & 0.0 & 0.0 & 0.0 \\
\hline 0.0 & 0.2 & 0.2 & 0.0 & 0.0 & 0.0 & 0.0 & 0.0 & 0.0 & 0.0 & 0.0 \\
\hline \multicolumn{2}{|c|}{ Total } & \multicolumn{2}{|c|}{138.2} & & & & & & & \\
\hline \multicolumn{2}{|c|}{$\log ($ sum $)$} & \multicolumn{2}{|c|}{2.1} & & & & & & & \\
\hline \multicolumn{2}{|c|}{$\log$} & \multicolumn{2}{|c|}{0.33052} & & & & & & & \\
\hline
\end{tabular}

Table 12. Forecast based on Bayesian model (Source: Author's data compilation [18] [19]).

\begin{tabular}{ccccccccccc}
\hline Location & 06 & $\mathbf{0 7}$ & $\mathbf{0 8}$ & $\mathbf{0 9}$ & $\mathbf{1 0}$ & $\mathbf{1 1}$ & $\mathbf{1 2}$ & $\mathbf{1 3}$ & $\mathbf{1 4}$ & $\mathbf{1 5}$ \\
\hline Equatorial Guinea & 0 & 0 & 1 & 0 & 0 & 0 & 0 & 0 & 0 & 0 \\
Ghana & 3 & 1 & 7 & 3 & 0 & 2 & 2 & 1 & 4 & 2 \\
Guinea & 4 & 2 & 0 & 5 & 6 & 5 & 3 & 1 & 0 & 3 \\
Guinea Bissau & 0 & 0 & 0 & 1 & 0 & 0 & 0 & 0 & 0 & 0 \\
Total & 7 & 3 & 8 & 9 & 6 & 7 & 5 & 2 & 4 & 5 \\
\hline & & & & Equatorial Guinea & & & \\
\hline-1.1334 & 0 & 0 & 1 & 0 & $\mathbf{0}$ & $\mathbf{0}$ & $\mathbf{0}$ & $\mathbf{0}$ & $\mathbf{0}$ & $\mathbf{0}$ \\
\hline
\end{tabular}




\section{Continued}

\begin{tabular}{|c|c|c|c|c|c|c|c|c|c|c|}
\hline 2.4736 & 0.0 & 0.0 & 2.5 & 0.0 & 0.0 & 0.0 & 0.0 & 0.0 & 0.0 & 0.0 \\
\hline-2.8068 & 0.0 & 0.0 & -2.8 & 0.0 & 0.0 & 0.0 & 0.0 & 0.0 & 0.0 & 0.0 \\
\hline 4.9233 & 0.0 & 0.0 & 4.9 & 0.0 & 0.0 & 0.0 & 0.0 & 0.0 & 0.0 & 0.0 \\
\hline-6.3929 & 0.0 & 0.0 & -6.4 & 0.0 & 0.0 & 0.0 & 0.0 & 0.0 & 0.0 & 0.0 \\
\hline \multirow[t]{3}{*}{ Total } & 0.0 & 0.0 & 2.9 & 0.0 & 0.0 & 0.0 & 0.0 & 0.0 & 0.0 & 0.0 \\
\hline & \multicolumn{10}{|c|}{ Ghana } \\
\hline & 3 & 1 & 7 & 3 & 0 & 2 & 2 & 1 & 4 & 2 \\
\hline-1.1334 & -3.4 & -1.1 & -7.9 & -3.4 & 0.0 & -2.3 & -2.3 & -1.1 & -4.5 & -2.3 \\
\hline 2.4736 & 7.4 & 2.5 & 17.3 & 7.4 & 0.0 & 4.9 & 4.9 & 2.5 & 9.9 & 4.9 \\
\hline-2.8068 & -8.4 & -2.8 & -19.6 & -8.4 & 0.0 & -5.6 & -5.6 & -2.8 & -11.2 & -5.6 \\
\hline 4.9233 & 14.8 & 4.9 & 34.5 & 14.8 & 0.0 & 9.8 & 9.8 & 4.9 & 19.7 & 9.8 \\
\hline-6.3929 & -19.2 & -6.4 & -44.8 & -19.2 & 0.0 & -12.8 & -12.8 & -6.4 & -25.6 & $5-12.8$ \\
\hline \multirow[t]{3}{*}{ Total } & 8.8 & 2.9 & 20.6 & 8.8 & 0.0 & 5.9 & 5.9 & 2.9 & 11.7 & 5.9 \\
\hline & \multicolumn{10}{|c|}{ Guinea } \\
\hline & 4 & 2 & 0 & 5 & 6 & 5 & 3 & 1 & 0 & 3 \\
\hline-1.1334 & -4.5 & -2.3 & 0.0 & -5.7 & -6.8 & -5.7 & -3.4 & -1.1 & -4.5 & -3.4 \\
\hline 2.4736 & 9.9 & 4.9 & 0.0 & 12.4 & 14.8 & 12.4 & 7.4 & 2.5 & 9.9 & 7.4 \\
\hline-2.8068 & -11.2 & -5.6 & 0.0 & -14.0 & -16.8 & -14.0 & -8.4 & -2.8 & -11.2 & -8.4 \\
\hline 4.9233 & 19.7 & 9.8 & 0.0 & 24.6 & 29.5 & 24.6 & 14.8 & 4.9 & 19.7 & 14.8 \\
\hline-6.3929 & -25.6 & -12.8 & 0.0 & -32.0 & -38.4 & -32.0 & -19.2 & -6.4 & -25.6 & $5-19.2$ \\
\hline \multirow[t]{3}{*}{ Total } & 11.7 & 5.9 & 0.0 & 14.7 & 17.6 & 14.7 & 8.8 & 2.9 & 11.7 & 8.8 \\
\hline & \multicolumn{10}{|c|}{ Guinea Bissau } \\
\hline & 0 & 0 & 0 & 1 & 0 & 0 & 0 & 0 & 0 & 0 \\
\hline-1.1334 & 0.0 & 0.0 & 0.0 & -1.1 & 0.0 & 0.0 & 0.0 & 0.0 & 0.0 & 0.0 \\
\hline 2.4736 & 0.0 & 0.0 & 0.0 & 2.5 & 0.0 & 0.0 & 0.0 & 0.0 & 0.0 & 0.0 \\
\hline-2.8068 & 0.0 & 0.0 & 0.0 & -2.8 & 0.0 & 0.0 & 0.0 & 0.0 & 0.0 & 0.0 \\
\hline 4.9233 & 0.0 & 0.0 & 0.0 & 4.9 & 0.0 & 0.0 & 0.0 & 0.0 & 0.0 & 0.0 \\
\hline-6.3929 & 0.0 & 0.0 & 0.0 & -6.4 & 0.0 & 0.0 & 0.0 & 0.0 & 0.0 & 0.0 \\
\hline \multirow[t]{3}{*}{ Total } & 0.0 & 0.0 & 0.0 & 2.9 & 0.0 & 0.0 & 0.0 & 0.0 & 0.0 & 0.0 \\
\hline & \multicolumn{10}{|c|}{ Total } \\
\hline & 7 & 3 & 8 & 9 & 6 & 7 & 5 & 2 & 4 & 5 \\
\hline-1.1334 & -7.9 & -3.4 & -9.1 & -10.2 & -6.8 & -7.9 & -5.7 & -2.3 & -4.5 & -5.7 \\
\hline 2.4736 & 17.3 & 7.4 & 19.8 & 22.3 & 14.8 & 17.3 & 12.4 & 4.9 & 9.9 & 12.4 \\
\hline-2.8068 & -19.6 & -8.4 & -22.5 & -25.3 & -16.8 & -19.6 & -14.0 & -5.6 & -11.2 & -14.0 \\
\hline 4.9233 & 34.5 & 14.8 & 39.4 & 44.3 & 29.5 & 34.5 & 24.6 & 9.8 & 19.7 & 24.6 \\
\hline-6.3929 & -44.8 & -19.2 & -51.1 & -57.5 & -38.4 & -44.8 & $-32.0-$ & -12.8 & -25.6 & $5-32.0$ \\
\hline \multirow[t]{2}{*}{ Total } & 20.6 & 8.8 & 23.5 & 26.4 & 17.6 & 20.6 & 14.7 & 5.9 & 11.7 & 14.7 \\
\hline & \multicolumn{10}{|c|}{ Predictor } \\
\hline Equatorial Guinea & 0.0 & 0.0 & 2.9 & 0.0 & 0.0 & 0.0 & 0.0 & 0.0 & 0.0 & 0.0 \\
\hline $\log$ & 0.0 & 0.0 & 0.5 & 0.0 & 0.0 & 0.0 & 0.0 & 0.0 & 0.0 & 0.0 \\
\hline Ghana & 8.8 & 2.9 & 20.6 & 8.8 & 0.0 & 5.9 & 5.9 & 2.9 & 11.7 & 5.9 \\
\hline
\end{tabular}




\section{Continued}

\begin{tabular}{ccccccccccc}
\hline Log & 0.9 & 0.5 & 1.3 & 0.9 & 0.0 & 0.8 & 0.8 & 0.5 & 1.1 & 0.8 \\
Guinea & 11.7 & 5.9 & 0.0 & 14.7 & 17.6 & 14.7 & 8.8 & 2.9 & 11.7 & 8.8 \\
Log & 1.1 & 0.8 & 0.0 & 1.2 & 1.2 & 1.2 & 0.9 & 0.5 & 1.1 & 0.9 \\
Guinea Bissau & 0.0 & 0.0 & 0.0 & 2.9 & 0.0 & 0.0 & 0.0 & 0.0 & 0.0 & 0.0 \\
Log & 0.0 & 0.0 & 0.0 & 0.5 & 0.0 & 0.0 & 0.0 & 0.0 & 0.0 & 0.0 \\
Total & 20.6 & 8.8 & 23.5 & 26.4 & 17.6 & 20.6 & 14.7 & 5.9 & 11.7 & 14.7 \\
Log & 1.3 & 0.9 & 1.4 & 1.4 & 1.2 & 1.3 & 1.2 & 0.8 & 1.1 & 1.2 \\
Sum of Logs & 29.1 & & & & & & & & \\
Ln(Sum of Logs) & 3.36996 & & & & & & & & \\
\hline
\end{tabular}

Table 13. Forecasted piracy attacks on gulf of Guinea (Source: Compilation from [18] [19]).

\begin{tabular}{lcccccc}
\hline & Equatorial Guinea & Ghana & Guinea & Guinea Bissau & Total & Average \\
\hline 2016 & 4 & 6 & 7 & 4 & 9 & 6 \\
2017 & 7 & 9 & 10 & 7 & 12 & 9 \\
2018 & 10 & 12 & 13 & 10 & 15 & 12 \\
2019 & 13 & 15 & 16 & 13 & 18 & 15 \\
2020 & 16 & 18 & 19 & 16 & 21 & 18 \\
2021 & 19 & 21 & 22 & 19 & 24 & 21 \\
2022 & 22 & 24 & 25 & 22 & 27 & 24 \\
2023 & 25 & 27 & 28 & 25 & 30 & 27 \\
2024 & 28 & 30 & 31 & 28 & 33 & 30 \\
2025 & 31 & 33 & 34 & 31 & 36 & 33 \\
2026 & 34 & 36 & 37 & 34 & 39 & 36 \\
2027 & 37 & 39 & 40 & 37 & 42 & 39 \\
2028 & 40 & 42 & 43 & 40 & 45 & 42 \\
2029 & 43 & 45 & 46 & 43 & 48 & 45 \\
2030 & 46 & 48 & 49 & 46 & 51 & 48 \\
\hline
\end{tabular}

\section{Forecasted Increase in Attacks}

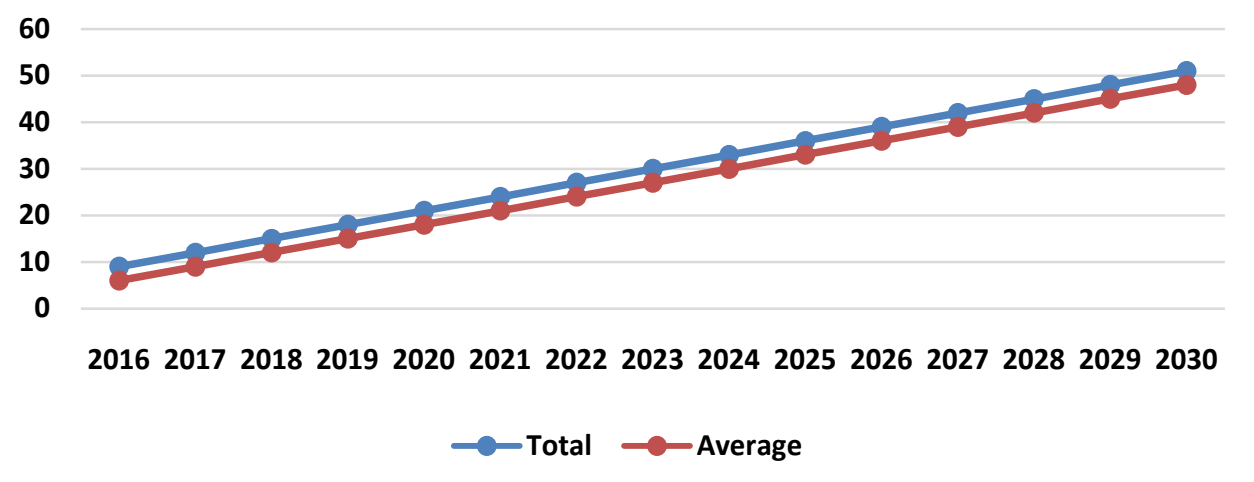

Figure 7. Projected increase in piracy attacks. Source: (Author's data compilation from [18] [19]). 
by their navies or coastguard. This will help alleviate or terminate any surprises which could be sprung on them by pirates that could disturb traffic flow.

\section{References}

[1] Treves, T. (2009) Piracy, Law of the Sea, and Use of Force: Developments off the Coast of Somalia. European Journal of International Law, 20, 399-414. https://doi.org/10.1093/ejil/chp027

[2] Besley, T., Fetzer, T. and Mueller, H. (2015) The Welfare Cost of Lawlessness; Evidence from Somali Piracy. Journal of the European Economic Association, 13, 203 239. https://doi.org/10.1111/jeea.12114

[3] Geiss, R. and Petrig, A. (2011) Piracy and Armed Robbery at Sea: The Legal Framework for Counter-Piracy Operations in Somalia and the Gulf of Aden. Vol. 1, Oxford University Press, Oxford. https://doi.org/10.1093/acprof:oso/9780199609529.001.0001

[4] Hansen, J., Jacobs, G., Hsu, L., Dykes, J., Dastugue, J., Allard, R., Mittu, R., et al. (2011) Information Domination: Dynamically Coupling METOC and INTEL for Improved Guidance for Interdiction. Naval Research Lab, Washington DC, 110 115.

[5] Boot, M. (2009) Pirates, Then and Now-How Piracy Was Defeated in the Past and Can Be Again. Foreign Affairs, 88, 94-107.

[6] Bäccman, C., Hjärthag, F. and Almqvist, K. (2016) Improved Resiliency and Well Being among Military Personnel in a Swedish Naval Force after a Counter Piracy Operation off the Coast of Somalia. Scandinavian Journal of Psychology, 57, 350358. https://doi.org/10.1111/sjop.12299

[7] Jean-Jacques, D.A. (2011) Somali Piracy and the Introduction of Somalia to the Western World. Doctoral Dissertation, University of Central Florida Orlando.

[8] Hu, S., Fang, Q., Xia, H. and Xi, Y. (2007) Formal Safety Assessment Based on Relative Risks Model in Ship Navigation. Reliability Engineering \& System Safety, 92, 369-377. https://doi.org/10.1016/j.ress.2006.04.011

[9] Zhou, H. and Seibel, K. (2015) Marine Insecurity in the Gulf of Guinea: A Greater Role for China? China Brief, 15.

[10] McNicholas, M. (2016) Maritime Security: An Introduction. Butterworth-Heinemann, Oxford.

[11] Marchione, E. and Johnson, S.D. (2013) Spatial, Temporal and Spatio-Temporal Patterns of Maritime Piracy. Journal of Research in Crime and Delinquency, 50, 504-524. https://doi.org/10.1177/0022427812469113

[12] Peretto, K. (2008) Weak States Off-Shore: Piracy in Modern Times. East African Human Security Forum, Nairobi.

[13] Nincic, D. (2009) Maritime Piracy in Africa: The Humanitarian Dimension. African Security Studies, 18, 1-16. https://doi.org/10.1080/10246029.2009.9627538

[14] Onuoha, F. (2009) Sea Piracy and Maritime Security in the Horn of Africa: The Somali Coast and Gulf of Aden in Perspective. African Security Studies, 18, 31-44. https://doi.org/10.1080/10246029.2009.9627540

[15] Hart, T. (2014) Gulf of Guinea: Two Pirate Tales. Risk Map Maritime. https://www.controlrisks.com/webcasts/studio/riskmap-maritime-2014/2014-02-24RiskMap_MARITIME-2014-gulf-of-guinea.pdf

[16] Benkenstein, A. (2013) West Africa: Gulf of Guinea-The New Flashpoint of Piracy in Africa? http://allafrica.com/stories/201312171230.html 
[17] Ghana Maritime Authority (2014) Commissioning of VTMIS Project. GMA, Accra.

[18] International Maritime Bureau (2010) Piracy and Armed Robbery against Ships: Annual Report.

[19] International Maritime Bureau (2015a) GISIS: Piracy and Armed Robbery. https://gisis.imo.org/Public/PAR/Reports.aspx?Name=RegionalAnalysis

[20] Bowden, A. (2010) The Economic Cost of Maritime Piracy. One Earth Future Foundation.

[21] Goerlandt, F. and Montewka, J. (2015) A Framework for Risk Analysis of Maritime Transportation Systems: A Case Study for Oil Spill from Tankers in a Ship-Ship Collision. Safety Science, 76, 42-66. https://doi.org/10.1016/j.ssci.2015.02.009

[22] Green, W.H. (2012) Econometric Analysis. 7th Edition, Pearson Education, Inc., Boston.

[23] Harrell, F.F. (2015) Ordinal Logistic Regression. In: Harrell, F.E., Ed., Springer Series in Statistics, Springer International Publishing, Cham, 311-325. https://doi.org/10.1007/978-3-319-19425-7_13

[24] Kleinbaum, D.G. and Klein, M. (2010) Ordinal Logistic Regression. In: Kleinbaum, D.G. and Klein, M., Eds., Logistic Regression, Springer, New York, 463-488. https://doi.org/10.1007/978-1-4419-1742-3_13

[25] McCullagh, P. (1980) Regression Models for Ordinal Data. Journal of the Royal Statistical Society. Series B (Methodological), 42, 109-142.

[26] Reyna, O.T. (2014) Predicted Probabilities and Marginal Effects after (Ordered) Logit/Probitusing Margins in Stata.

[27] Jiang, B. (2014) Maritime Piracy in Malacca, Strait and South China Sea: Testing the Deterrence and Reactance Models. University of Pennsylvania and University of Maryland, Baltimore.

[28] Dugan, L. (2011) The Series Hazard Model: An Alternative to Time Series for Event Data. Journal of Quantitative Criminology, 27, 379-402.

https://doi.org/10.1007/s10940-010-9127-1

[29] Chen, X., Minacapelli, L., Fishman, S., Orehek, E., Dechesne, M., Segal, E. and Kruglanski, A.W. (2008) Impact of Political and Military Interventions on Terrorist Activities in Gaza and the West Bank: A Hazard Modelling Analysis.

[30] LaFree, G., Dugan, L. and Korte, R. (2009) Is Counter Terrorism Counterproductive? Northern Ireland 1969-1992. Criminology, 47, 501-530.

[31] Aven, T. (2008) Risk Analysis: Assessing Uncertainties beyond Expected Values and Probabilities. Wiley \& Sons, Chichester. https://doi.org/10.1002/9780470694435

[32] Fenton, N. and Neil, M. (2012) Risk Assessment and Decision Analysis with Bayesian Networks. CRC Press, Boca Raton.

[33] Goerlandt, F. and Montewka, J. (2015) A Framework for Risk Analysis of Maritime Transportation Systems: A Case Study for Oil Spill from Tankers in a Ship-Ship Collision. Safety Science, 76, 42-66. https://doi.org/10.1016/j.ssci.2015.02.009

[34] Montewka, J., Ehlers, S., Goerlandt, F., Hinz, T., Tabri, K. and Kujala, P. (2014) A Framework for Risk Assessment for Maritime Transportation Systems-A Case Study for Open Sea Collisions Involving RoPax Vessels. Relability Engineering \& System Safety, 124, 142-157. https://doi.org/10.1016/j.ress.2013.11.014

[35] Hänninen, M., Mazaheri, A., Kujala, P., Montewka, J., Laaksonen, P., Salmiovitre, M. and Klang, M. (2013) Expert Elicitation of a Navigation Service Implementation Effects on Ship Groundings and Collisions in the Gulf of Finland. Proceedings of the Institution of Mechanical Engineers, Part O: Journal of Risk and Reliability, 228, 
19-28.

[36] Koller, D. and Friedman, N. (2009) Probabilistic Graphical Models: Principle and Techniques. MIT Press, Cambridge.

Submit or recommend next manuscript to SCIRP and we will provide best service for you:

Accepting pre-submission inquiries through Email, Facebook, LinkedIn, Twitter, etc. A wide selection of journals (inclusive of 9 subjects, more than 200 journals)

Providing 24-hour high-quality service

User-friendly online submission system

Fair and swift peer-review system

Efficient typesetting and proofreading procedure

Display of the result of downloads and visits, as well as the number of cited articles Maximum dissemination of your research work

Submit your manuscript at: http://papersubmission.scirp.org/

Or contact jss@scirp.org 